\title{
Kinetic Resolution of Propargylic alcohols Catalyzed by Benzotetramisole
}

Vladimir B. Birman* and Lei Guo

A contribution from the Department of Chemistry, Washington University, Campus Box 1134, One Brookings Drive, Saint Louis, Missouri 63130

SUPPORTING INFORMATION: Experimental procedures (7 pages), ${ }^{1} \mathrm{H}$ NMR and ${ }^{13} \mathrm{C}$ NMR spectra (24 pages).

\section{EXPERIMENTAL PART.}

1. GENERAL. All reagents were obtained commercially and used as received unless otherwise specified. Catalysts $\mathbf{2}$ and $\mathbf{3}$ were prepared as previously described. Substrate $\mathbf{9 a}$ is commercially available from Sigma-Aldrich. All the other substrates are known compounds and have been prepared according to the literature procedures: $4 \mathbf{a}-\mathbf{d},{ }^{2 \mathrm{c}} 5,{ }^{11} \mathbf{6},{ }^{2 \mathrm{c}} 7,{ }^{12} \mathbf{8 a},{ }^{9 \mathrm{a}} \mathbf{8 b}, \mathbf{c}^{13} \mathbf{9 b}{ }^{14}$.

${ }^{1} \mathrm{H}$ NMR and ${ }^{13} \mathrm{C}$ NMR spectra were recorded on a Unity 300 or a Unity $500 \mathrm{MHz}$ Varian spectrometer. The chemical shifts are reported as $\delta$ values (ppm) relative to TMS using residual $\mathrm{CHCl}_{3}$ peak (7.26 ppm) as the reference. High-Resolution mass spectral analyses were performed at Washington University MS Center on a Kratos MS-50TA spectrometer using electron ionization (HR-EI) and fast atom bombardment (FAB) methods. Optical rotations were measured in dichloromethane at room temperature and $589 \mathrm{~nm}$ wavelength using a Perkin-Elmer 241 polarimeter.

\section{KINETIC RESOLUTION EXPERIMENTS}

Figure 1S. Catalysts and substrates used in this study

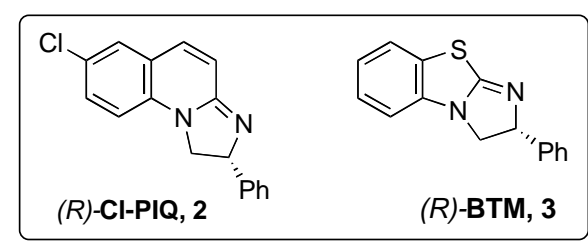

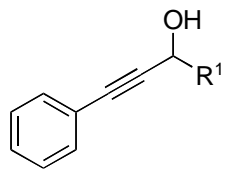

4a: $\mathrm{R}^{1}=\mathrm{Me}$

4b: $R^{1}=E t$

4c: $\mathrm{R}^{1}=i-\mathrm{Pr}$

4d: $R^{1}=t-B u$

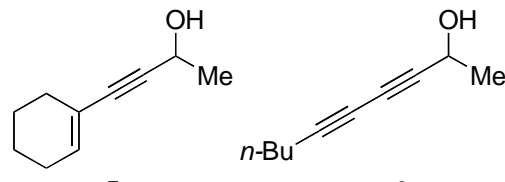

5

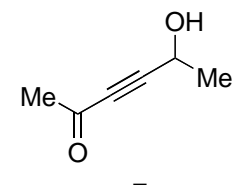

7

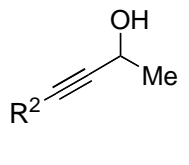

8a: $\mathrm{R}^{2}=n-\mathrm{Bu}$
8b: $\mathrm{R}^{2}=$ Cyclohexyl
8c: $\mathrm{R}^{2}=t-\mathrm{Bu}$ 8d: $R^{2}=H$ 


\subsection{Optimization of reaction conditions}

Procedure A: The first test. The procedure described earlier ${ }^{5 \mathrm{c}}$ was followed. Each of the stock catalyst solutions was prepared by dissolving $0.010 \mathrm{mmol}$ of the respective catalyst [(R)-Cl-PIQ 2 or (R)-BTM 3] and $66 \mathrm{mg}(0.38 \mathrm{mmol})$ of $N, N$-diisopropylethylamine in $\mathrm{CDCl}_{3}$ in a $1.00 \mathrm{~mL}$ volumetric test tube and bringing the volume to the mark. Two one dram vials were each charged with $37 \mathrm{mg}(0.25 \mathrm{mmol})$ of 4-phenyl-3-butyn-2-ol, $0.50 \mathrm{~mL}$ of the stock solution of the catalyst and $100 \mathrm{mg}$ of dry granular $\mathrm{Na}_{2} \mathrm{SO}_{4}$, cooled to $0^{\circ} \mathrm{C}$ and treated with $24 \mu \mathrm{L}(0.19 \mathrm{mmol})$ of propionic anhydride. The temperature was maintained at $0^{\circ} \mathrm{C}$. The reaction was monitored by ${ }^{1} \mathrm{H}$ NMR and stopped by pouring the contents into a vial with $\mathrm{MeOH}$ upon reaching conversion about $50 \%$. The workup and HPLC analysis followed the standard procedure. ${ }^{5}$ The results are shown in Table $1 \mathrm{~S}$.

Procedure B: Base-free conditions. Procedure A was followed except that no base was added.

Procedure C: The use of isobutyric anhydride. Procedure B was followed except propionic anhydride was replaced with isobutyric anhydride.

Procedure D: Variation of the solvent. Procedure B was followed except $\mathrm{CDCl}_{3}$ was replaced with THF and tert-amyl alcohol, respectively.

Table 1S. Optimization of reaction conditions

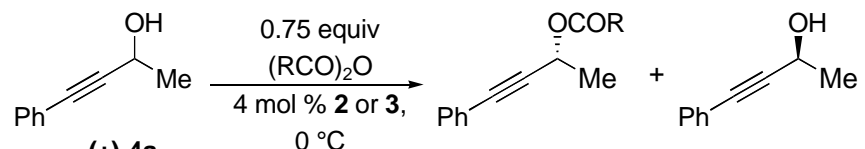

$( \pm)-4 a$

$0{ }^{\circ} \mathrm{C}$

\begin{tabular}{|c|c|c|c|c|c|c|c|c|c|c|c|c|}
\hline entry & catalyst & anhydride & base & solvent & $\begin{array}{c}\text { time, } \\
\mathbf{h} \\
\end{array}$ & \# & $\begin{array}{c}\mathbf{e e}_{\mathrm{E}} \\
\%\end{array}$ & $\begin{array}{c}\mathbf{e e}_{\mathrm{A}} \\
\%\end{array}$ & $\begin{array}{c}\mathrm{C}_{\mathrm{HPLC}} \\
\%\end{array}$ & $\mathbf{s}$ & $\begin{array}{c}\mathrm{C}_{\mathrm{AVG}} \\
\% \\
\end{array}$ & $\begin{array}{c}\mathbf{S}_{\mathrm{AV}} \\
\mathrm{G}\end{array}$ \\
\hline \multirow{2}{*}{1} & \multirow{2}{*}{2} & \multirow{2}{*}{$(\mathrm{EtCO})_{2} \mathrm{O}$} & \multirow{2}{*}{$i-\mathrm{Pr}_{2} \mathrm{NEt}$} & \multirow{2}{*}{$\mathrm{CDCl}_{3}$} & \multirow{2}{*}{1.0} & 1 & 74.8 & 83.6 & 52.8 & 17.9 & \multirow{2}{*}{52} & \multirow{2}{*}{19} \\
\hline & & & & & & 2 & 77.1 & 81.0 & 51.2 & 19.1 & & \\
\hline \multirow{2}{*}{2} & \multirow{2}{*}{3} & \multirow{2}{*}{$(\mathrm{EtCO})_{2} \mathrm{O}$} & \multirow{2}{*}{$i-\mathrm{Pr}_{2} \mathrm{NEt}$} & \multirow{2}{*}{$\mathrm{CDCl}_{3}$} & \multirow{2}{*}{5.0} & 1 & 81.3 & 89.2 & 52.3 & 28.8 & \multirow{2}{*}{52} & \multirow{2}{*}{28} \\
\hline & & & & & & 2 & 80.4 & 89.1 & 52.6 & 27.3 & & \\
\hline \multirow{2}{*}{3} & \multirow{2}{*}{2} & \multirow{2}{*}{$(\mathrm{EtCO})_{2} \mathrm{O}$} & \multirow{2}{*}{ none } & \multirow{2}{*}{$\mathrm{CDCl}_{3}$} & \multirow{2}{*}{45.5} & 1 & 80.0 & 57.0 & 41.6 & 15.9 & \multirow{2}{*}{41} & \multirow{2}{*}{18} \\
\hline & & & & & & 2 & 83.3 & 56.7 & 40.5 & 19.4 & & \\
\hline \multirow{2}{*}{4} & \multirow{2}{*}{3} & \multirow{2}{*}{$(\mathrm{EtCO})_{2} \mathrm{O}$} & \multirow{2}{*}{ none } & \multirow{2}{*}{$\mathrm{CDCl}_{3}$} & \multirow{2}{*}{10.5} & 1 & 69.0 & 99.5 & 59.0 & 30.7 & \multirow{2}{*}{59} & \multirow{2}{*}{31} \\
\hline & & & & & & 2 & 68.5 & 99.6 & 59.3 & 30.6 & & \\
\hline \multirow{2}{*}{5} & \multirow{2}{*}{2} & \multirow{2}{*}{$(i-\mathrm{PrCO})_{2} \mathrm{O}$} & \multirow{2}{*}{ none } & & & 1 & 74.4 & 39.3 & 34.6 & 10.0 & & \\
\hline & & & & $\mathrm{CDCH}_{3}$ & 32 & 2 & 74.0 & 38.7 & 34.3 & 9.7 & 34 & 10 \\
\hline$C_{1} \rightarrow$ & & (:D $=\mathrm{CP})$ & & & & 1 & 81.2 & 70.2 & 46.4 & 20.1 & 17 & 21 \\
\hline 0 & J & $(\mathrm{I}-\mathrm{HICU})_{2} \mathrm{U}$ & Mone & $\mathrm{CDCl}_{3}$ & 21.0 & 2 & 81.8 & 71.8 & 46.7 & 21.3 & $4 /$ & 21 \\
\hline
\end{tabular}




\begin{tabular}{|c|c|c|c|c|c|c|c|c|c|c|c|c|}
\hline \multirow{2}{*}{7} & \multirow{2}{*}{2} & \multirow{2}{*}{$(\mathrm{EtCO})_{2} \mathrm{O}$} & \multirow{2}{*}{ none } & \multirow{2}{*}{ THF } & \multirow{2}{*}{6.5} & 1 & 77.2 & 65.9 & 46.1 & 15.3 & \multirow{2}{*}{46} & \multirow{2}{*}{15} \\
\hline & & & & & & 2 & 77.6 & 65.7 & 45.8 & 15.6 & & \\
\hline \multirow{2}{*}{8} & \multirow{2}{*}{3} & \multirow{2}{*}{$(\mathrm{EtCO})_{2} \mathrm{O}$} & \multirow{2}{*}{ none } & \multirow{2}{*}{ THF } & \multirow{2}{*}{24} & 1 & - & - & - & - & \multirow{2}{*}{ NR } & \multirow{2}{*}{-} \\
\hline & & & & & & 2 & - & - & - & - & & \\
\hline \multirow{2}{*}{9} & \multirow{2}{*}{2} & \multirow{2}{*}{$(\mathrm{EtCO})_{2} \mathrm{O}$} & \multirow{2}{*}{ none } & $t$-amyl & \multirow{2}{*}{7.0} & 1 & 80.3 & 44.3 & 35.6 & 14.1 & \multirow{2}{*}{37} & \multirow{2}{*}{14} \\
\hline & & & & alcohol & & 2 & 78.1 & 48.0 & 38.1 & 13.0 & & \\
\hline \multirow{2}{*}{10} & \multirow{2}{*}{3} & \multirow{2}{*}{$(\mathrm{EtCO})_{2} \mathrm{O}$} & \multirow{2}{*}{ none } & $t$-amyl & \multirow{2}{*}{7.0} & 1 & 77.9 & 69.8 & 47.3 & 16.7 & \multirow{2}{*}{47} & \multirow{2}{*}{17} \\
\hline & & & & alcohol & & 2 & 77.8 & 70.2 & 47.4 & 16.6 & & \\
\hline
\end{tabular}

2.2. Variation of the substrates. Procedure B described above was followed in all cases, except that increased catalyst loadings (Table 2S, entries 5 and 8-12) and propionic anhydride concentrations (entry 5) were employed for slow-reacting substrates.

Table 5S. Kinetic resolution of propargylic alcohols

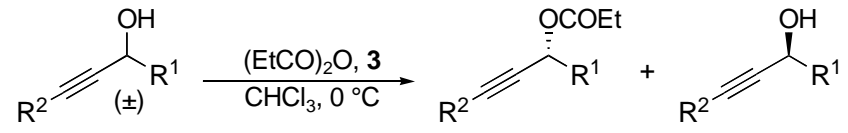

\begin{tabular}{|c|c|c|c|c|c|c|c|c|c|c|}
\hline entry & substrate & $\begin{array}{l}\text { catalyst } \\
\text { loading }\end{array}$ & $\begin{array}{c}\text { time, } \\
\text { h }\end{array}$ & \# & $\begin{array}{c}\mathbf{e e}_{\mathrm{E}} \\
\%\end{array}$ & $\begin{array}{c}\mathbf{e e}_{\mathrm{A}} \\
\%\end{array}$ & $\begin{array}{c}\mathrm{C}_{\text {HPLC }} \\
\%\end{array}$ & $\mathbf{S}$ & $\begin{array}{c}\mathrm{C}_{\mathrm{AVG}} \\
\%\end{array}$ & $\mathbf{S}_{\mathrm{AVC}}$ \\
\hline \multirow{2}{*}{1} & \multirow{2}{*}{$4 a$} & \multirow{2}{*}{$4 \mathrm{~mol} \%$} & \multirow{2}{*}{10.5} & 1 & 69.1 & 99.5 & 59.0 & 30.7 & \multirow{2}{*}{59} & \multirow{2}{*}{31} \\
\hline & & & & 2 & 68.5 & 99.6 & 59.3 & 30.6 & & \\
\hline \multirow{2}{*}{2} & \multirow{2}{*}{$4 b$} & \multirow{2}{*}{$4 \mathrm{~mol} \%$} & \multirow{2}{*}{10.5} & 1 & 75.4 & 95.8 & 56.0 & 27.1 & \multirow{2}{*}{56} & \multirow{2}{*}{27} \\
\hline & & & & 2 & 75.2 & 96.4 & 56.2 & 27.6 & & \\
\hline \multirow{2}{*}{3} & \multirow{2}{*}{$4 c$} & \multirow{2}{*}{$4 \mathrm{~mol} \%$} & \multirow{2}{*}{10.5} & 1 & 71.4 & 89.8 & 55.7 & 17.8 & \multirow{2}{*}{56} & \multirow{2}{*}{18} \\
\hline & & & & 2 & 71.3 & 90.6 & 56.0 & 18.2 & & \\
\hline \multirow{2}{*}{4} & \multirow{2}{*}{ 4d } & \multirow{2}{*}{$4 \mathrm{~mol} \%$} & \multirow{2}{*}{10.5} & 1 & 69.7 & 53.0 & 43.2 & 9.4 & \multirow{2}{*}{53} & \multirow{2}{*}{43} \\
\hline & & & & 2 & 70.1 & 53.7 & 43.4 & 9.6 & & \\
\hline \multirow{2}{*}{5} & \multirow{2}{*}{5} & \multirow{2}{*}{$10 \mathrm{~mol} \%$} & \multirow{2}{*}{18} & 1 & 61.9 & 99.8 & 61.7 & 26.7 & \multirow{2}{*}{62} & \multirow{2}{*}{27} \\
\hline & & & & 2 & 63.0 & 99.8 & 61.3 & 27.5 & & \\
\hline \multirow{2}{*}{6} & \multirow{2}{*}{6} & \multirow{2}{*}{$4 \mathrm{~mol} \%$} & 20 & 1 & 83.3 & 88.7 & 51.6 & 32.3 & רז & רת \\
\hline & & & 2.0 & 2 & 82.3 & 89.7 & 52.1 & 31.1 & 52 & 32 \\
\hline 7 & 7 & 1 & $1-$ & 1 & 77.5 & 92.8 & 54.5 & 26.1 & $\tau \Gamma$ & r \\
\hline 7 & 7 & $4 \mathrm{~mol} \%$ & 1.5 & 2 & 77.7 & 93.1 & 54.5 & 26.7 & 55 & 26 \\
\hline 0 & 0 & 10 & ז & 1 & 76.7 & 54.5 & 41.5 & 13.0 & 17 & 12 \\
\hline 8 & $8 a$ & $10 \mathrm{~mol} \%$ & 25 & 2 & 76.0 & 54.1 & 41.6 & 12.5 & 42 & 13 \\
\hline 0 & $0 \mathbf{b}$ & 10 & כרי & 1 & 59.6 & 89.7 & 60.1 & 11.5 & $c 0$ & 11 \\
\hline 9 & $8 D$ & $10 \mathrm{~mol} \%$ & 23 & 2 & 58.4 & 87.6 & 60.0 & 10.4 & bU & 11 \\
\hline 10 & 0 & 10 & 10 & 1 & 59.7 & 55.4 & 48.1 & 6.8 & 40 & 7 \\
\hline 10 & $8 \mathrm{C}$ & $10 \mathrm{~mol} \%$ & 19 & 2 & 60.0 & 55.6 & 48.1 & 6.9 & 48 & 7 \\
\hline
\end{tabular}




\begin{tabular}{|c|c|c|c|c|c|c|c|c|c|c|}
\hline \multirow{2}{*}{11} & \multirow{2}{*}{$9 \mathbf{a}$} & \multirow{2}{*}{$10 \mathrm{~mol} \%$} & \multirow{2}{*}{6.0} & 1 & 63.2 & 77.9 & 55.2 & 10.2 & \multirow{2}{*}{55} & \multirow{2}{*}{11} \\
\cline { 5 - 8 } & & & 2 & 67.0 & 80.9 & 54.7 & 12.3 & & \\
\hline \multirow{2}{*}{12} & \multirow{2}{*}{$9 \mathbf{9}$} & \multirow{2}{*}{$10 \mathrm{~mol} \%$} & \multirow{2}{*}{2.5} & 1 & 49.7 & 63.2 & 56.0 & 5.5 & \multirow{2}{*}{57} & \multirow{2}{*}{5} \\
\cline { 5 - 7 } & & & 2 & 47.5 & 65.1 & 57.8 & 5.3 & & \\
\hline
\end{tabular}

4. HPLC analyses. HPLC analyses were performed on a Shimadzu LC system using UV detection at 254 or $204 \mathrm{~nm}$. Most propargylic alcohols were separated on Chiralcel OD-H analytical chiral stationary phase column (4.6x250 mm, Chiral Technologies, Inc.) using hexane-isopropanol mixtures as mobile phase. Most esters of propargylic alcohols were hydrolyzed with methanolic $\mathrm{NaOH}$ to the alcohols, which were analyzed by HPLC. All exceptions are noted in the footnotes to Table 3S.

5. Absolute stereochemistry. The absolute configurations of the unreacted propargylic obtained by KR were assigned by comparison of the sign of optical rotation with the literature data or via the Mosher ester analysis ${ }^{15}$.

Table 3S. HPLC and optical rotation data

\begin{tabular}{|c|c|c|c|c|c|}
\hline \multirow[b]{2}{*}{ Substrate } & \multirow{2}{*}{$\begin{array}{c}\text { HPLC } \\
\text { conditions }\end{array}$} & \multicolumn{2}{|c|}{ Retention time (min) } & \multicolumn{2}{|c|}{ Unreacted alcohol } \\
\hline & & (R)-ent & (S)-ent & $\begin{array}{l}\text { Sign of } \\
\text { rotation }\end{array}$ & $\begin{array}{c}\text { Absolute } \\
\text { Configuration }\end{array}$ \\
\hline $4 a$ & $\begin{array}{c}15 \% \\
\text { IPA/hexane } \\
\text { OD-H }\end{array}$ & 6.76 & 12.30 & $(-)$ & $\mathrm{S}^{9 \mathrm{a}}$ \\
\hline $4 \mathbf{b}$ & $\begin{array}{c}12 \% \\
\text { IPA/hexane } \\
\text { OD-H }\end{array}$ & 7.38 & 15.42 & $(-)$ & $S^{10 e}$ \\
\hline $4 c$ & $\begin{array}{c}10 \% \\
\text { IPA/hexane } \\
\text { OD-H }\end{array}$ & 6.64 & 10.83 & $(-)$ & $S^{10 \mathrm{e}}$ \\
\hline 4d & $\begin{array}{c}6 \% \\
\text { IPA/hexane } \\
\text { OD-H }\end{array}$ & 8.03 & 10.56 & $(-)$ & $\mathrm{S}^{16}$ \\
\hline 5 & $\begin{array}{c}6 \% \\
\text { IPA/hexane } \\
\text { OD-H }\end{array}$ & 7.06 & 7.97 & N/D & $S^{a}$ \\
\hline 6 & $\begin{array}{c}2 \% \\
\text { IPA/hexane } \\
\text { OD-H }\end{array}$ & 17.91 & 19.31 & $(-)$ & $S^{2 c}$ \\
\hline
\end{tabular}




\begin{tabular}{|c|c|c|c|c|c|}
\hline $7^{b}$ & $\begin{array}{c}0.5 \% \\
\text { IPA/hexane } \\
\text { OD-H }\end{array}$ & 15.40 & 16.60 & N/D & $\mathrm{S}^{\mathrm{a}}$ \\
\hline $8 a$ & $\begin{array}{c}1 \% \\
\text { IPA/hexane } \\
\text { OD-H } \\
\end{array}$ & 18.05 & 20.30 & $(-)$ & $S^{9 a}$ \\
\hline $8 \mathbf{b}$ & $\begin{array}{c}2 \% \\
\text { IPA/hexane } \\
\text { OD-H }\end{array}$ & 10.45 & 12.30 & $(-)$ & $\mathrm{S}^{13}$ \\
\hline $8 c^{c}$ & $\begin{array}{c}6 \% \\
\text { IPA/hexane } \\
\text { OD-H }\end{array}$ & 10.05 & 11.87 & $(-)$ & $S^{13}$ \\
\hline 9a & $\begin{array}{c}2 \% \\
\text { IPA/hexane } \\
\text { OD-H }\end{array}$ & 16.70 & 15.60 & $(-)$ & $S^{17}$ \\
\hline $9 b^{d}$ & $\begin{array}{c}2 \% \\
\text { IPA/hexane } \\
\text { OD-H }\end{array}$ & 16.70 & 15.60 & $(-)$ & $S^{10 e, 18}$ \\
\hline
\end{tabular}

a) Absolute configuration was assigned by Mosher ester analysis ${ }^{15}$

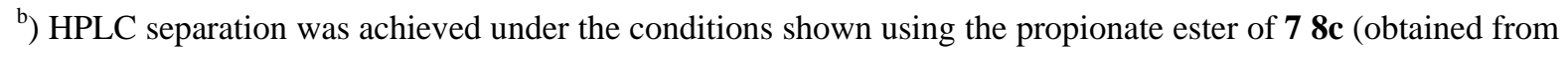
the alcohol by treatment with (EtCO) $)_{2} \mathrm{O}$ and DMAP).

$\left.{ }^{c}\right)$ HPLC separation was achieved under the conditions shown using the phenylcarbamate of $\mathbf{8 c}$ (obtained from the alcohol by treatment with PhNCO and DMAP).

${ }^{d}$ ) HPLC separation was achieved under the conditions shown using $\mathbf{9 a}$ (obtained from $\mathbf{9 b}$ and its propionate by treatment with $\mathrm{NaOH} / \mathrm{MeOH}$ )

\section{Spectral data for previously unreported compounds.}

Propionate of 4a: ${ }^{1} \mathbf{H}$ NMR (300MHz, $\left.\mathrm{CDCl}_{3}\right)$ : $\delta$ 7.41-7.46 (m, 2H), 7.28-7.31 (m, 3H), 5.71(q, $J=5.7 \mathrm{~Hz}, 1 \mathrm{H}), 2.37(\mathrm{q}, J=5.1 \mathrm{~Hz}, 2 \mathrm{H}), 1.57(\mathrm{~d}, J=3.6 \mathrm{~Hz}, 3 \mathrm{H}), 1.17(\mathrm{t}, J=8.1 \mathrm{~Hz}, 3 \mathrm{H}) ;{ }^{13} \mathbf{C ~ N M R}$ (75MHz, $\left.\mathrm{CDCl}_{3}\right): \delta 173.52,132.08,128.78,128.45,122.57,87.81,84.69,60.82,27.86,21.75$, 9.22; IR (film, $\mathbf{~ c m}^{-1}$ ) 2987, 2940, 1742, 1177; MS: HR-EI calculated for $\mathrm{C}_{13} \mathrm{H}_{14} \mathrm{O}_{2} \mathrm{~m} / \mathrm{z}$ : 202.0994, found 202.0987.

Propionate of 4b: ${ }^{1} \mathbf{H}$ NMR (300MHz, $\left.\mathrm{CDCl}_{3}\right)$ : $\delta$ 7.43-7.46 (m, 2H), 7.26-7.31 (m, 3H), 5.78(q, $J=5.7 \mathrm{~Hz}, 1 \mathrm{H}$ ), 2.39 (q, $J=7.2 \mathrm{~Hz}, 2 \mathrm{H}$ ), 1.88 (quintet, $J=6.6 \mathrm{~Hz}, 2 \mathrm{H}), 1.18$ (t, $J=7.5 \mathrm{~Hz}, 3 \mathrm{H}$ ), 1.07 (t, $J=7.5 \mathrm{~Hz}, 3 \mathrm{H}) ;{ }^{13} \mathbf{C}$ NMR (75MHz, $\left.\mathbf{C D C l}_{3}\right): \delta 173.65,132.11,128.73,128.45,122.66,86.78$, 85.40, 65.66, 28.48, 27.87, 9.64, 9.28; IR (film, $\mathbf{~ c m}^{-1}$ ) 2975, 2940, 1743, 1176; MS: HR-EI calculated for $\mathrm{C}_{14} \mathrm{H}_{16} \mathrm{O}_{2} \mathrm{~m} / \mathrm{z}: 216.1150$, found 216.1142 . 
Propionate of 4c: ${ }^{1} \mathbf{H}$ NMR (300MHz, $\left.\mathrm{CDCl}_{3}\right): \delta$ 7.43-7.46 (m,2H), 7.26-7.32 (m, 3H), 5.47 (d, $J=4.5 \mathrm{~Hz}, 1 \mathrm{H}$ ), 2.40(q, $J=6.3 \mathrm{~Hz}, 2 \mathrm{H}), 2.09$ (sextet, $J=6.9 \mathrm{~Hz}, 1 \mathrm{H}), 1.18(\mathrm{t}, J=7.5 \mathrm{~Hz}, 3 \mathrm{H}), 1.08(\mathrm{~d}$, $J=9.3 \mathrm{~Hz}, 3 \mathrm{H}), 1.06(\mathrm{~d}, J=9.6 \mathrm{~Hz}, 3 \mathrm{H}) ;{ }^{13} \mathrm{C} \mathrm{NMR}\left(75 \mathrm{MHz}, \mathrm{CDCl}_{3}\right): \delta 173.65,132.11,128.70$, 128.44, 122.75, 85.91, 85.72, 69.41, 32.92, 27.89, 18.51, 17.88, 9.36; IR (film, cm ${ }^{-1}$ ) 2968, 2940, 2875, 1742, 1177; MS: HR-EI calculated for $\mathrm{C}_{15} \mathrm{H}_{18} \mathrm{O}_{2} \mathrm{~m} / \mathrm{z}: 230.1307$, found 230.1303.

Propionate of 4d: ${ }^{1} \mathbf{H}$ NMR (300MHz, $\left.\mathrm{CDCl}_{3}\right): \delta$ 7.43-7.46 (m, 2H), 7..29-7.31 (m, 3H), 5.37 (s, 1H), 2.41(q, $J=7.5 \mathrm{~Hz}, 2 \mathrm{H}), 1.19$ (t, $J=9.0 \mathrm{~Hz}, 3 \mathrm{H}), 1.08$ (s, 9H); ${ }^{13} \mathrm{C}$ NMR (75MHz, $\left.\mathrm{CDCl}_{3}\right): 173.68,132.10,128.65,128.43,122.83,85.94,85.75,72.26,35.71,27.90,25.90,9.36$; IR (film, $\mathbf{c m}^{-1}$ ) 2971, 2871, 1740, 1172; MS: HR-EI calculated for $\mathrm{C}_{16} \mathrm{H}_{20} \mathrm{O}_{2} \mathrm{~m} / \mathrm{z}: 244.1463$, found 244.1458.

Propionate of 5: ${ }^{1} \mathbf{H}$ NMR (300MHz, $\mathrm{CDCl}_{3}$ ): $\delta 6.13$ ( borad m, 1H), 5.59( q, J=6.6Hz, 1H), 2.31 (q, $J=7.5 \mathrm{~Hz}, 2 \mathrm{H}), 2.15-2.04$ (broad $\mathrm{m}, 4 \mathrm{H}), 1.70-1.51$ (broad $\mathrm{m}, 4 \mathrm{H}), 1.48$ ( d, $J=5.1 \mathrm{~Hz}, 3 \mathrm{H}$ ), 1.14 $(\mathrm{t}, J=7.2 \mathrm{~Hz}, 3 \mathrm{H}) ;{ }^{13} \mathrm{C}$ NMR $\left(75 \mathrm{MHz}, \mathrm{CDCl}_{3}\right): \delta 173.51,136.03,120.12,86.49,85.07,60.93$, 29.22, 27.85, 25.81, 22.42, 21.89, 21.63, 9.19; IR (film, $\mathbf{~ c m}^{-1}$ ) 2959, 2936, 1742, 1185, 1164; MS: HR-EI calculated for $\mathrm{C}_{13} \mathrm{H}_{18} \mathrm{O}_{2} \mathrm{~m} / \mathrm{z}: 206.1307$, found 207.1310 .

Propionate of 6: ${ }^{1} \mathbf{H}$ NMR (300MHz, $\left.\mathrm{CDCl}_{3}\right): \delta 5.45(\mathrm{q}, J=6.6,1 \mathrm{H}), 2.35(\mathrm{t}, J=7.8 \mathrm{~Hz}, 2 \mathrm{H}), 2.25(\mathrm{t}$, $J=7.2 \mathrm{~Hz}, 2 \mathrm{H}), 1.51-1.35$ (m, 4H), 1.46 ( d, $J=9.9 \mathrm{~Hz}, 3 \mathrm{H}), 1.11(\mathrm{t}, J=7.5 \mathrm{~Hz}, 3 \mathrm{H}), 0.88(\mathrm{t}, J=7.2 \mathrm{~Hz}$, $3 \mathrm{H}) ;{ }^{13} \mathbf{C}$ NMR (75MHz, $\left.\mathrm{CDCl}_{3}\right): \delta 173.29,82.18,73.78,69.90,64.49,60.51 ;$ IR (film, $\mathbf{~ c m}^{-\mathbf{1}}$ ) 2937, 2873, 1745, 1173; MS: HR-EI calculated for $\mathrm{C}_{13} \mathrm{H}_{18} \mathrm{O}_{2} \mathrm{~m} / \mathrm{z}$ : 206.1307, found 207.1343.

Propionate of 7: ${ }^{1} \mathbf{H}$ NMR (300MHz, $\left.\mathrm{CDCl}_{3}\right): \delta 5.52(\mathrm{q}, J=6.6 \mathrm{~Hz}, 1 \mathrm{H}), 2.33(\mathrm{q}, J=7.5 \mathrm{~Hz}, 2 \mathrm{H})$, 2.31 (s, 3H), 1.50 ( d, $J=6.9 \mathrm{~Hz}, 3 \mathrm{H}), 1.12$ (t, $J=7.5 \mathrm{~Hz}, 3 \mathrm{H}) ;{ }^{13} \mathbf{C}$ NMR $\left(75 \mathrm{MHz}, \mathrm{CDCl}_{3}\right): \delta 184.02$, 173.22, 89.14, 83.64, 59.50, 32.69, 27.58, 20.60, 9.05; IR (film, cm$^{-1}$ ) 2989, 2943, 1746, 1684, 1360, 1226, 1171; MS: HR-FAB calculated for $\mathrm{C}_{9} \mathrm{H}_{12} \mathrm{O}_{3}\left(\mathrm{M}+\mathrm{Li}^{+}\right) \mathrm{m} / \mathrm{z}$ : 175.0946 , found 175.0937.

Propionate of 8a: ${ }^{1} \mathbf{H}$ NMR (300MHz, $\mathrm{CDCl}_{3}$ ): $\delta 5.45$ (q, $\left.J=2.4 \mathrm{~Hz}, 1 \mathrm{H}\right), 2.33$ (q, $J=7.5 \mathrm{~Hz}, 2 \mathrm{H}$ ), 2.19 ( t, $J=7.2 \mathrm{~Hz}, 2 \mathrm{H}), 1.56-1.35$ (m, 4H), 1.42 (d, $J=6.2 \mathrm{~Hz}, 3 \mathrm{H}), 1.12$ (t, $J=7.8 \mathrm{~Hz}, 3 \mathrm{H}), 0.88$ (t, $J=7.2 \mathrm{~Hz}, 3 \mathrm{H}) ;{ }^{13} \mathrm{C}$ NMR $\left(75 \mathrm{MHz}, \mathrm{CDCl}_{3}\right): \delta 173.56,85.59,78.91,60.82,30.75,27.85,22.07$, 22.02, 18.53, 13.72, 9.18; IR (film, $\mathbf{~ c m}^{-\mathbf{1}}$ ) 2936, 1742, 1185, 1164; MS: HR-EI calculated for $\mathrm{C}_{11} \mathrm{H}_{18} \mathrm{O}_{2} \mathrm{~m} / \mathrm{z}: 182.1307$, found 182.1314 .

Propionate of 8b: ${ }^{1} \mathbf{H}$ NMR (300MHz, $\left.\mathrm{CDCl}_{3}\right): \delta 5.46$ (q, J=5.1Hz, 1H), $2.35(\mathrm{~m}, 1 \mathrm{H}), 2.31$ (q, $J=7.2 \mathrm{~Hz}, 2 \mathrm{H}$ ), 1.80-1.60 (m, 4H), 1.54-1.20( m, 6H), 1.43 (d, J=6.6Hz, 3H), 1.12 (t, $J=7.5 \mathrm{~Hz}, 3 \mathrm{H}$ ); ${ }^{13} \mathrm{C}$ NMR (75MHz, $\mathrm{CDCl}_{3}$ ): $\delta 173.55,89.57,78.93,60.85,32.66,29.13,27.90,26.05,24.97$, 22.14, 9.21; IR (film, $\mathbf{c m}^{-1}$ ) 2932, 1742, 1165; MS: HR-EI calculated for $\mathrm{C}_{13} \mathrm{H}_{20} \mathrm{O}_{2} \mathrm{~m} / \mathrm{z}$ : 208.1463, found 208.1487. 
Propionate of 8c: ${ }^{1} \mathbf{H}$ NMR (300MHz, $\left.\mathrm{CDCl}_{3}\right): \delta 5.46$ (q, $\left.J=6.6 \mathrm{~Hz}, 1 \mathrm{H}\right), 2.31(\mathrm{q}, J=7.5 \mathrm{~Hz}, 2 \mathrm{H})$, 1.41 ( d, $J=6.6 \mathrm{~Hz}, 3 \mathrm{H}), 1.19$ (s, 9H), 1.12 (t, $J=7.5 \mathrm{~Hz}, 3 \mathrm{H}) ;{ }^{13} \mathrm{C}$ NMR $\left(75 \mathrm{MHz}, \mathrm{CDCl}_{3}\right): \delta 173.51$, 93.63, 77.39, 60.76, 31.04, 27.91, 27.46, 22.15, 9.21; IR (film, $\mathbf{~ c m}^{-1}$ ) 2971, 2869, 1743, 1181; MS: HR-EI calculated for $\mathrm{C}_{11} \mathrm{H}_{18} \mathrm{O}_{2} \mathrm{~m} / \mathrm{z}: 182.1307$, found 182.1291 .

Phenylcarbamate of 8c: ${ }^{1} \mathbf{H}$ NMR $\left(300 \mathrm{MHz}, \mathrm{CDCl}_{3}\right): \delta 7.28-7.40(\mathrm{~m}, 4 \mathrm{H}), 7.06(\mathrm{t}, J=6.6 \mathrm{~Hz}, 1 \mathrm{H})$, 6.68 (broad s, 1H), 5.53 (q, $J=6.6 \mathrm{~Hz}, 1 \mathrm{H}), 1.51$ (d, $J=6.6 \mathrm{~Hz}, 3 \mathrm{H}), 1.23$ (s, 9H); ${ }^{13} \mathbf{C}$ NMR $(75 \mathrm{MHz}$, $\left.\mathrm{CDCl}_{3}\right): \delta 152.66,138.05,129.20,123.60,118.88,94.11,77.31,62.04,31.04,27.48,22.49$; IR (film, $\mathbf{~ c m}^{-1}$ ) 3320, 2970, 1703, 1600, 1542, 1226, 1051; MS: HR-EI calculated for $\mathrm{C}_{15} \mathrm{H}_{19} \mathrm{NO}_{2}$ $\mathrm{m} / \mathrm{z}: 245.1416$,found 245.1423 .

Propionate of 9b: ${ }^{1} \mathbf{H}$ NMR (300MHz, $\left.\mathrm{CDCl}_{3}\right): \delta 5.37(\mathrm{t}, J=5.1 \mathrm{~Hz}, 1 \mathrm{H}), 1.78-1.65(\mathrm{~m}, 2 \mathrm{H})$, 1.50-1.12 (m, 4H), 1.12 (t, $J=7.2 \mathrm{~Hz}, 3 \mathrm{H}), 0.87$ (t, $J=6.0 \mathrm{~Hz}, 3 \mathrm{H}), 0.14(\mathrm{~s}, 9 \mathrm{H}) ;{ }^{13} \mathrm{C}$ NMR $(75 \mathrm{MHz}$, $\left.\mathrm{CDCl}_{3}\right): \delta 173.42,103.23,90.19,90.19,64.37,34.97,31.39,27.81,24.78,22.60,14.06,9.20$; IR (film, $\mathbf{~ c m}^{-1}$ ) 2958, 2863, 1745, 1250, 1176, 844; MS: HR-EI calculated for $\mathrm{C}_{13} \mathrm{H}_{24} \mathrm{O}_{2} \mathrm{Si} \mathrm{m} / \mathrm{z}$ : 254.1702, found 254.1699.

\section{AdDitional RefERences}

${ }^{11}$ Schwier, T.; Rubin, M.; Gevorgyan, V. Org. Lett. 2004, 6, 1999.

${ }^{12}$ Li, P.; Fong, W. M.; Chao, L. C. F.; Fung, S. H. C.; Williams, I. D. J. Org. Chem. 2001, 66, 4087.

${ }^{13} \mathrm{Ng}$, S.; Jamison, T. J. Am. Chem. Soc. 2005, 127, 7320.

${ }^{14}$ Brinkmeyer, R. S.; Macdonald, T. L. J. Chem. Soc., Chem. Commun. 1978, 876.

${ }^{15}$ (a) Dale, J. A.; Dull, D.L.; Mosher, H. S. J. Org. Chem., 1969, 34, 2543; (b) Dale, J. A.; Mosher, H, S. J. Am. Chem. Soc. 1973, 95, 512; (c) Ohtani, I.; Kusumi, T.; Kashman, Y.; Kakisawa, H. J. Am. Chem. Soc. 1991, 113, 4092.

${ }^{16}$ Ramachandran, P. V.; Teodorovic, A. V.; Rangaishenvi, M. V.; Brown, H. C. J. Org. Chem. 1992, 57, 2379.

${ }^{17}$ S-(-)-1-octyn-3-ol, Aldrich catalog 2005-2006, p. 1779. 


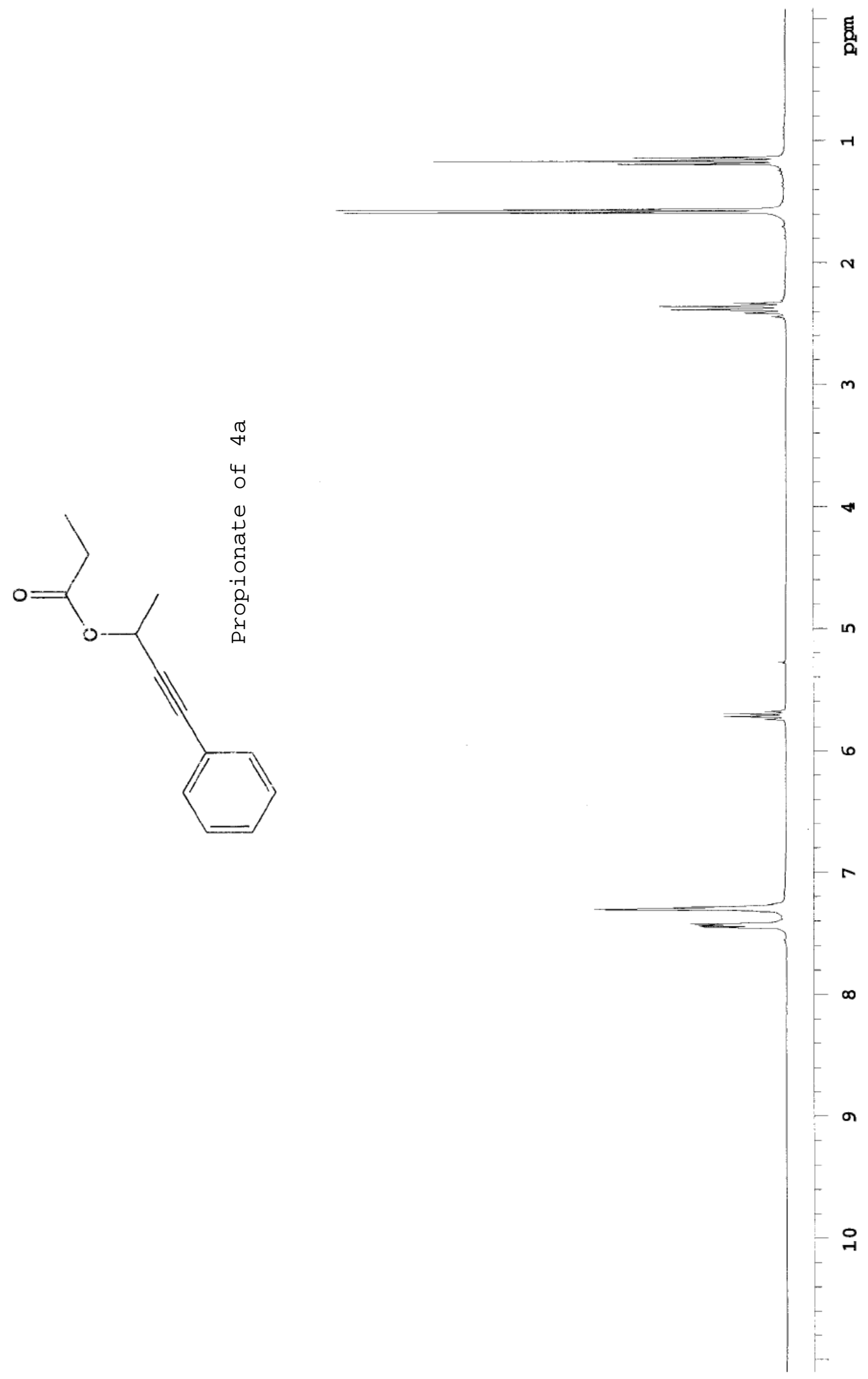




$$
1
$$




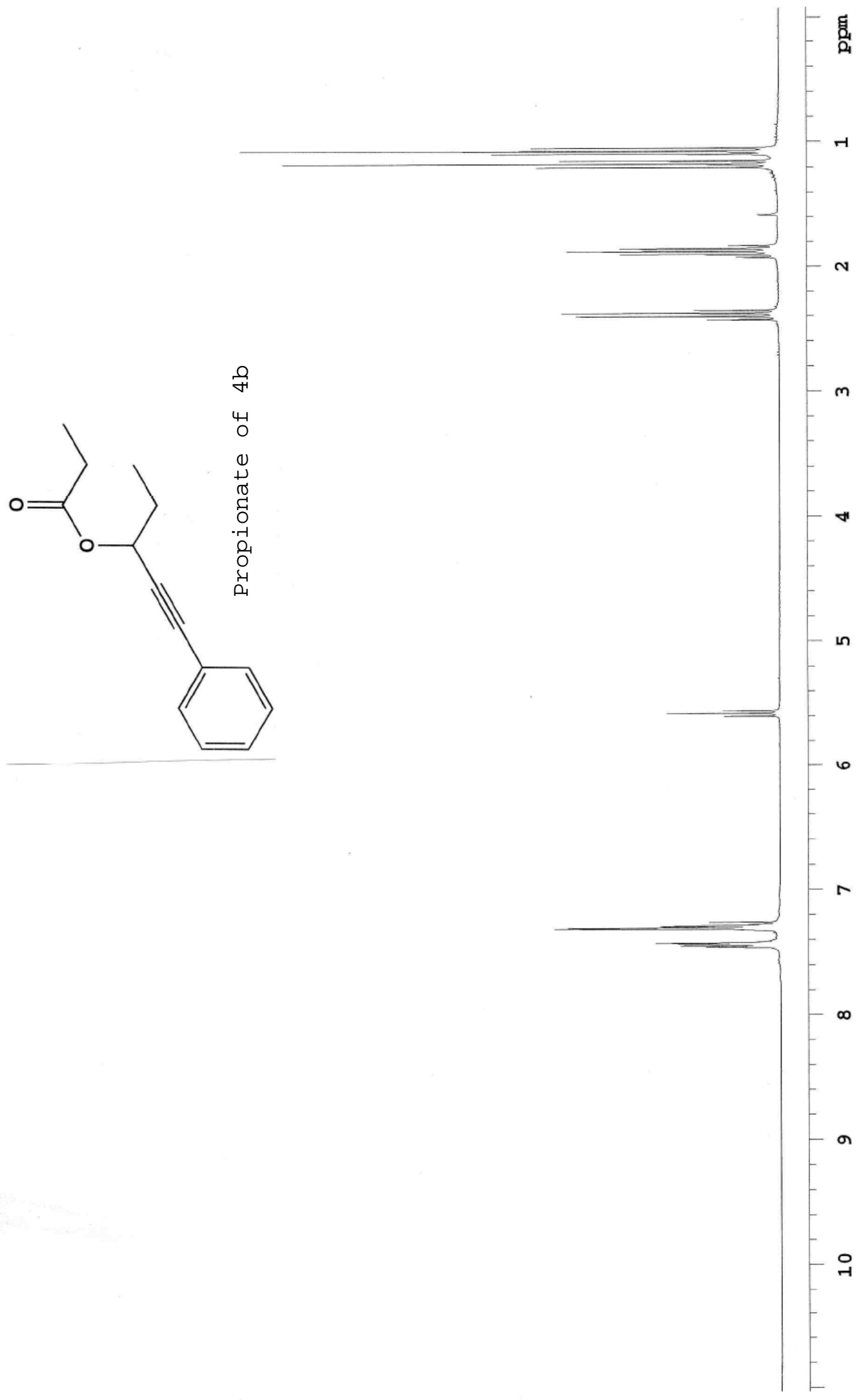



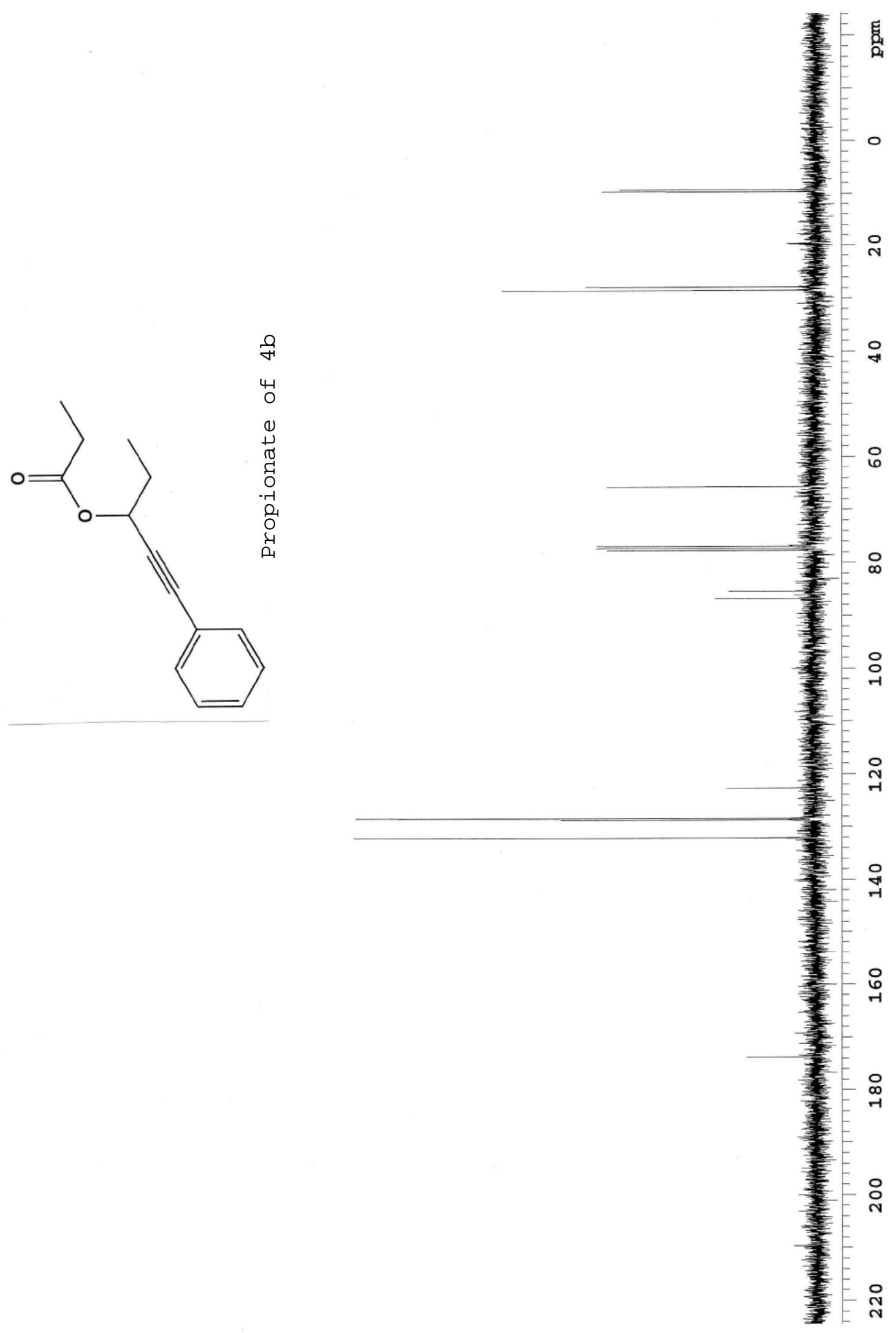


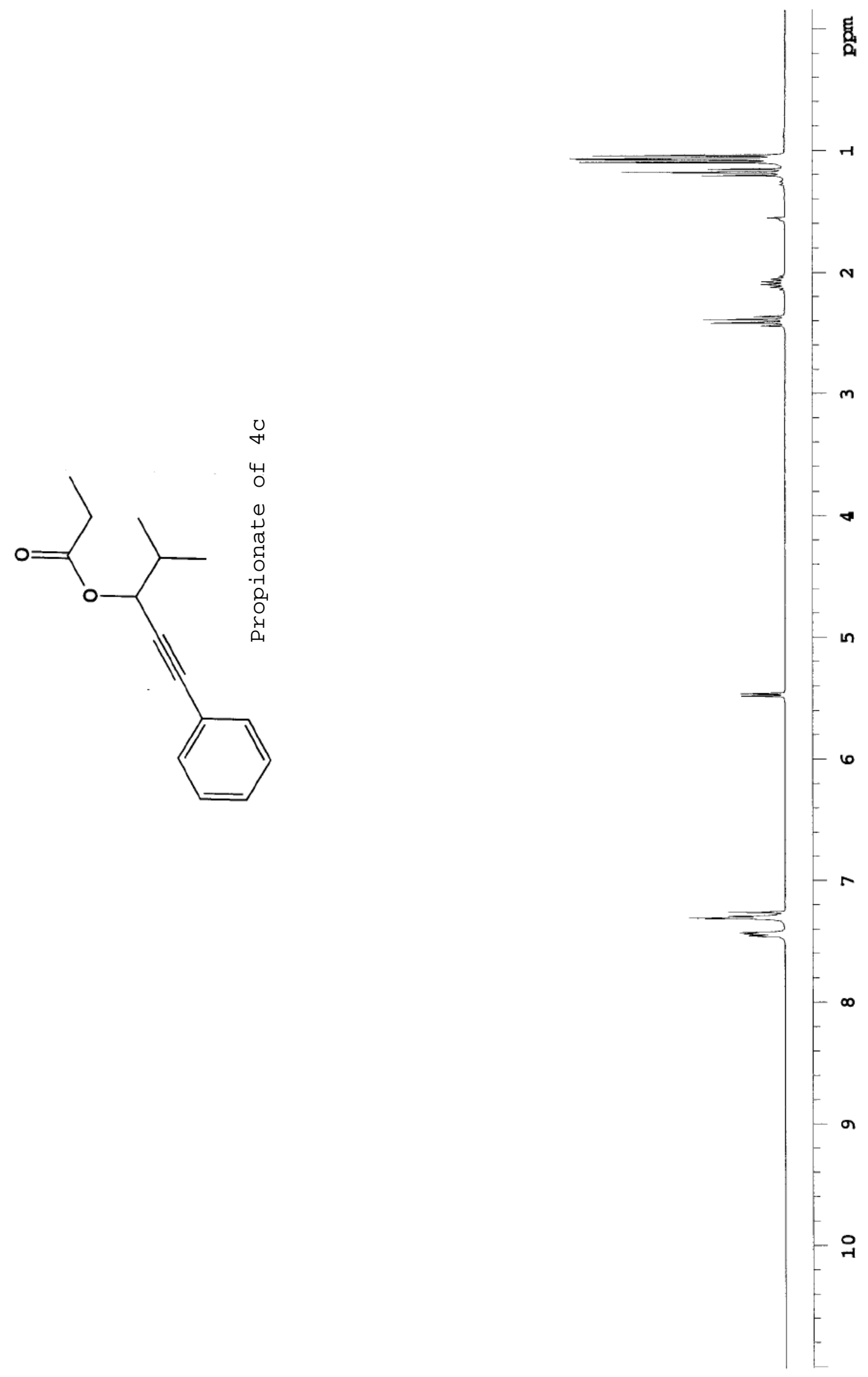



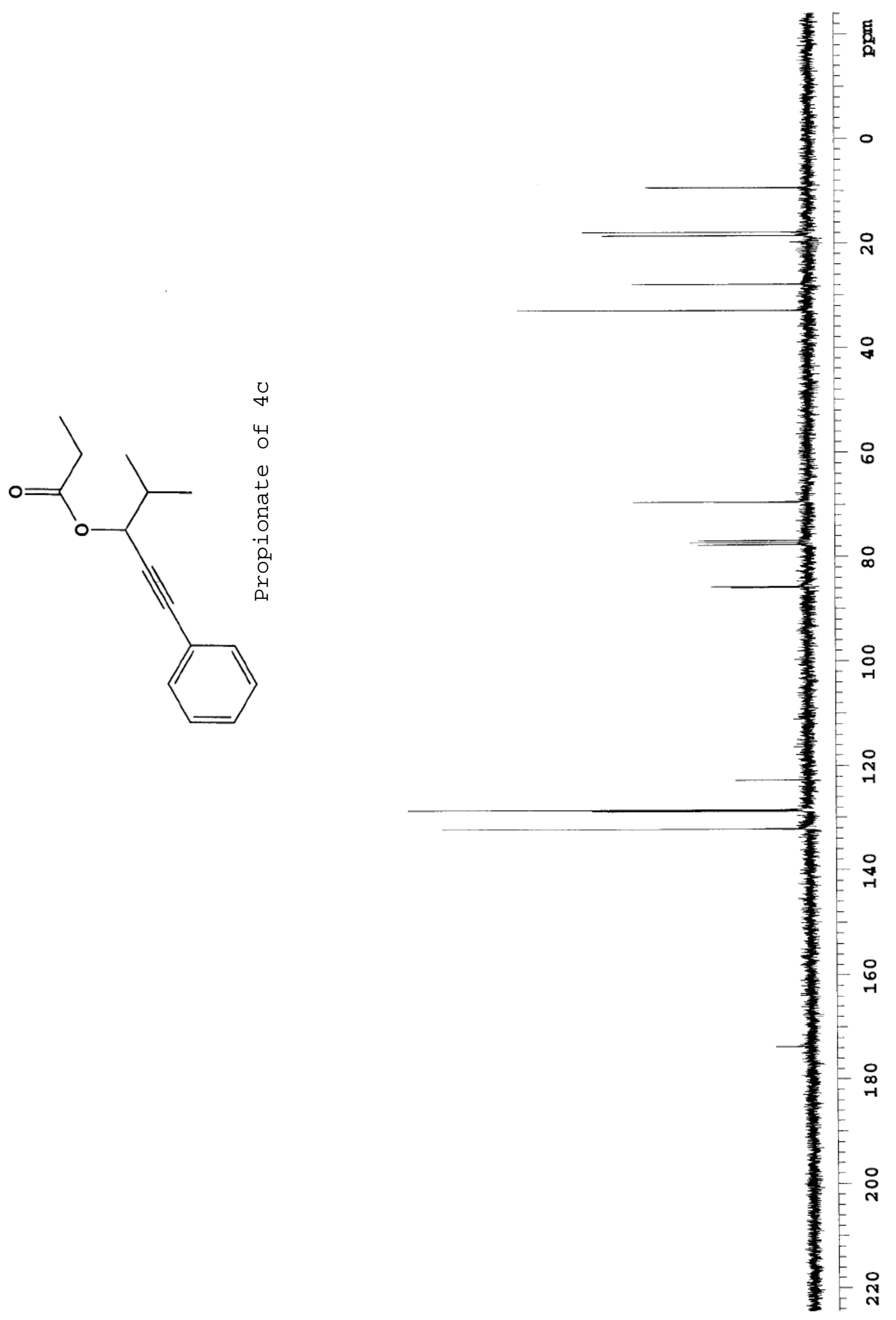


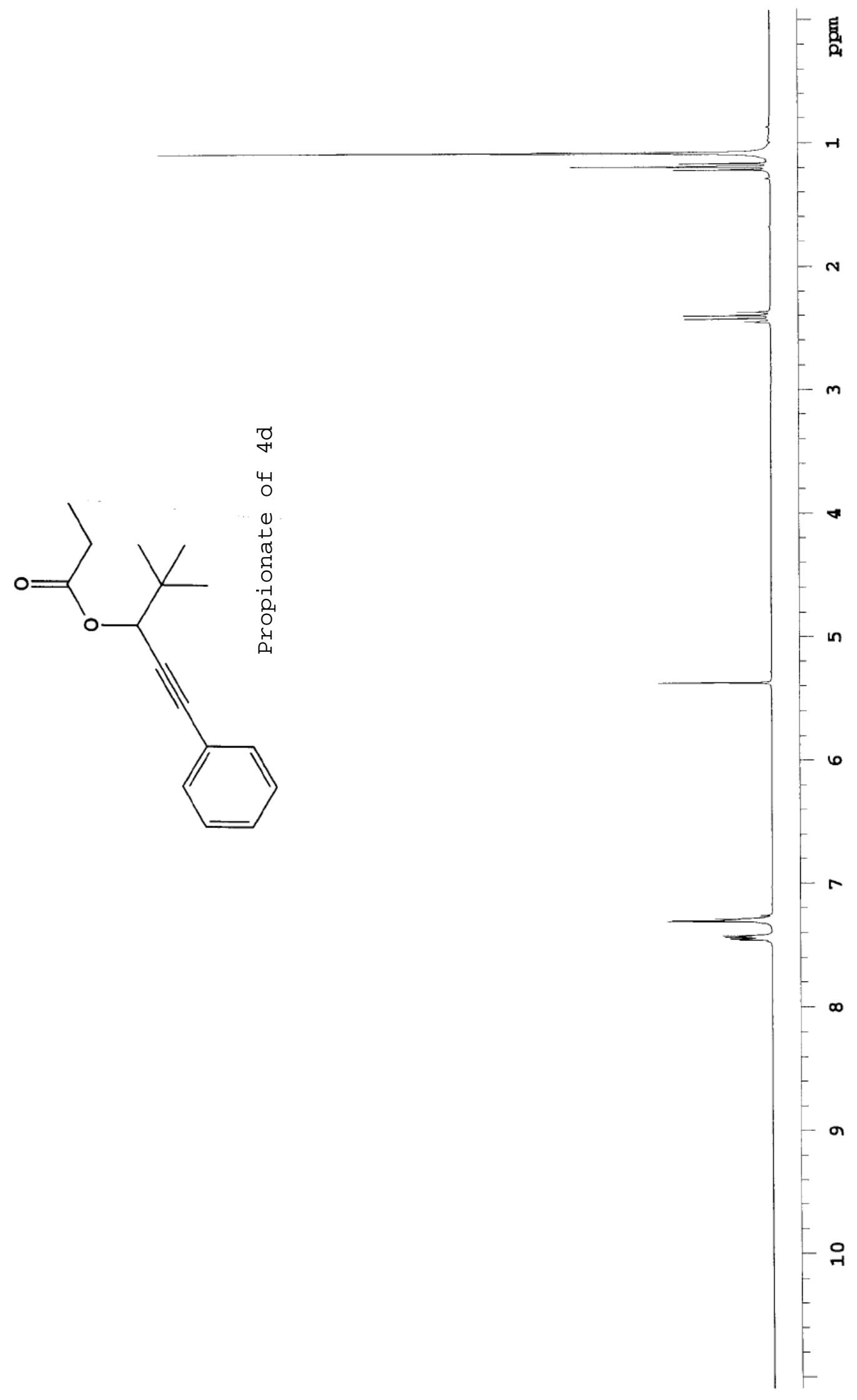




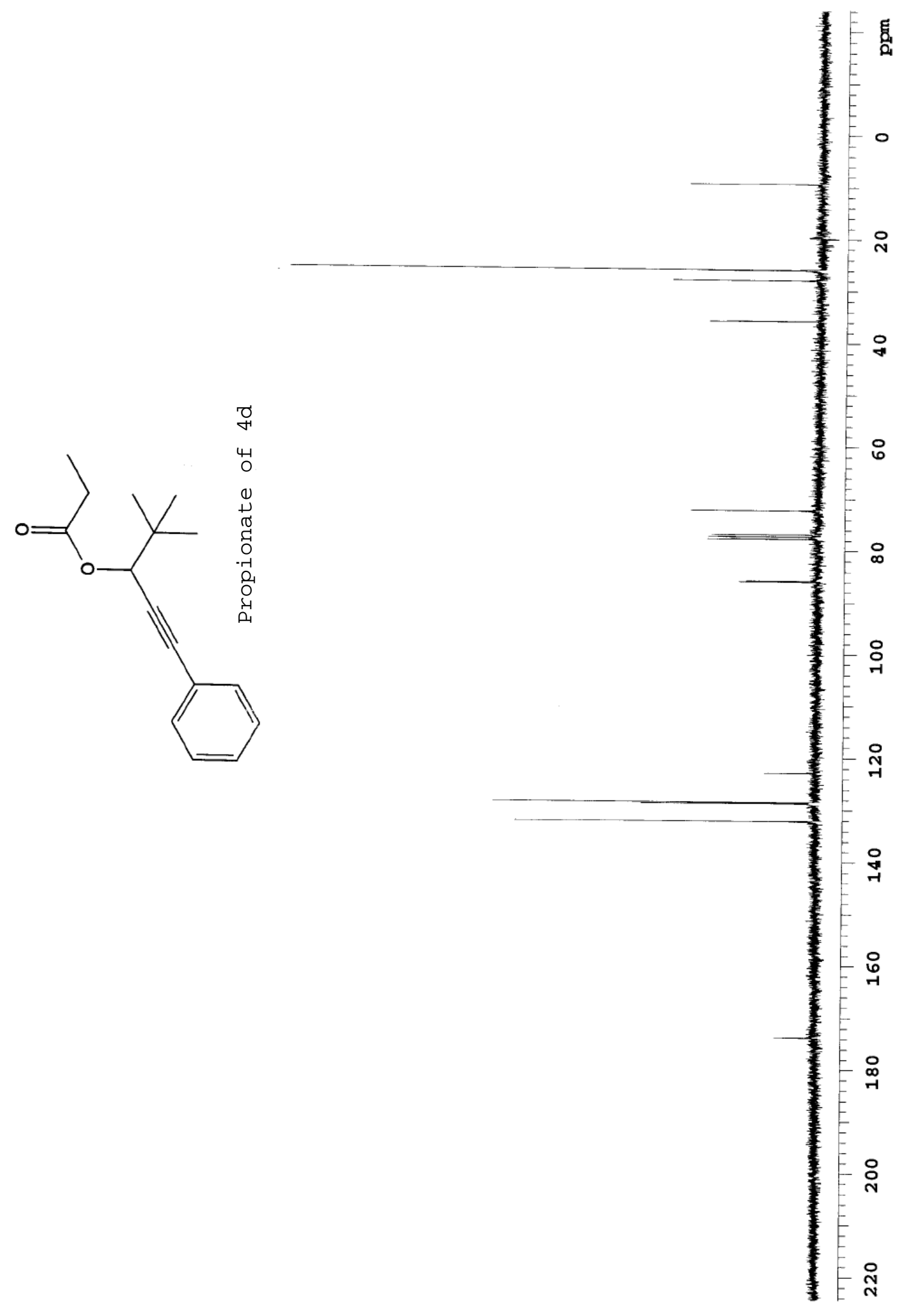




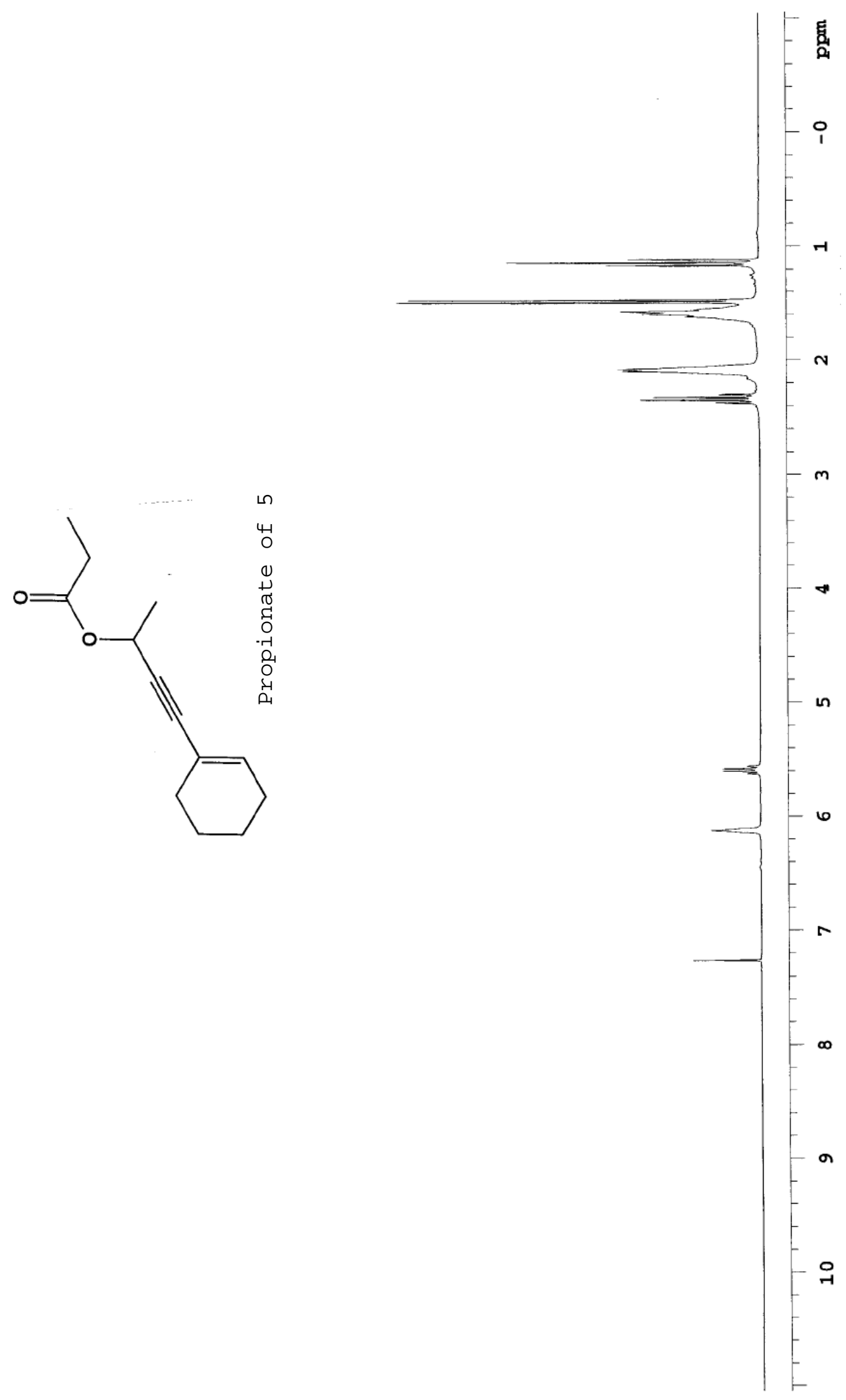



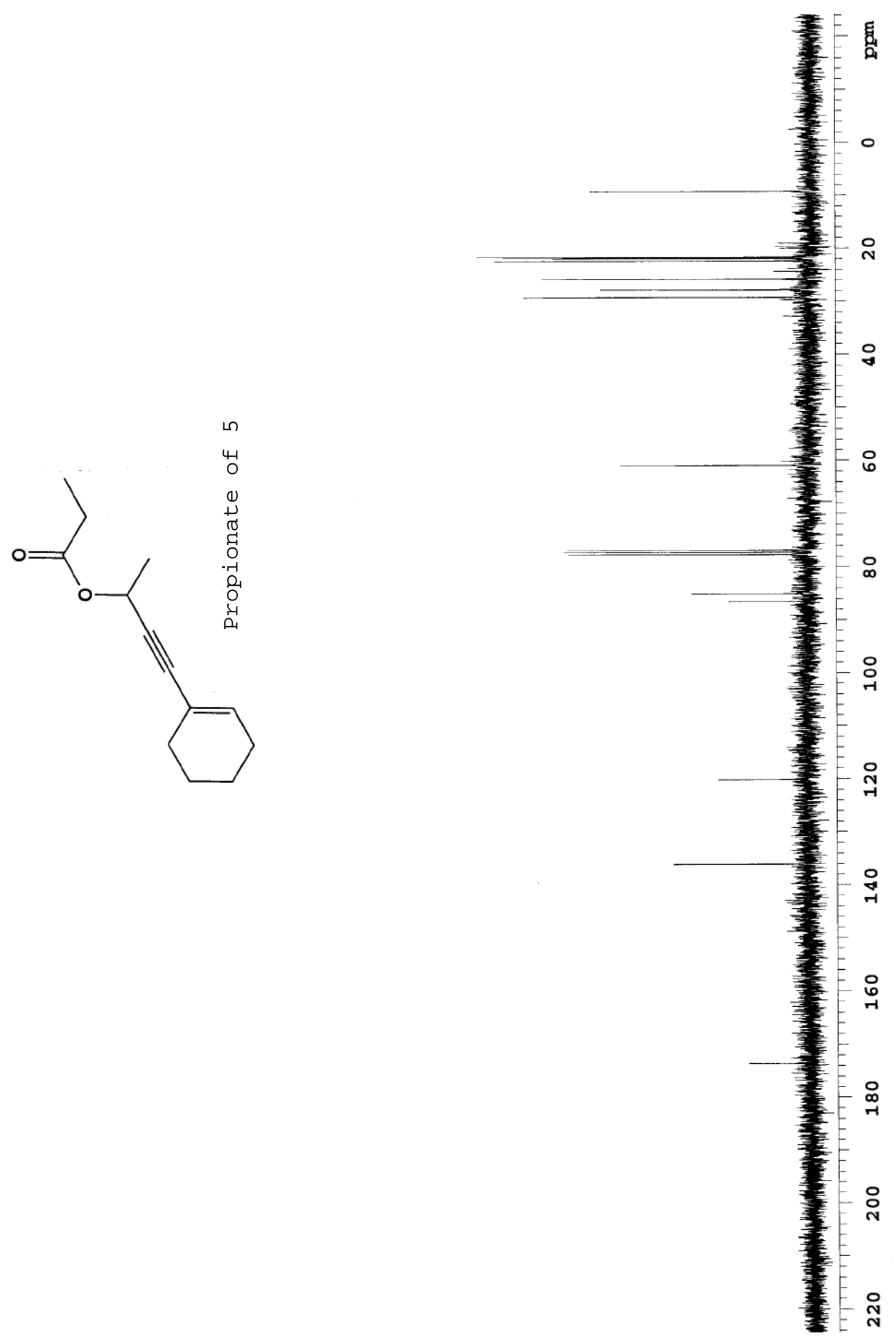


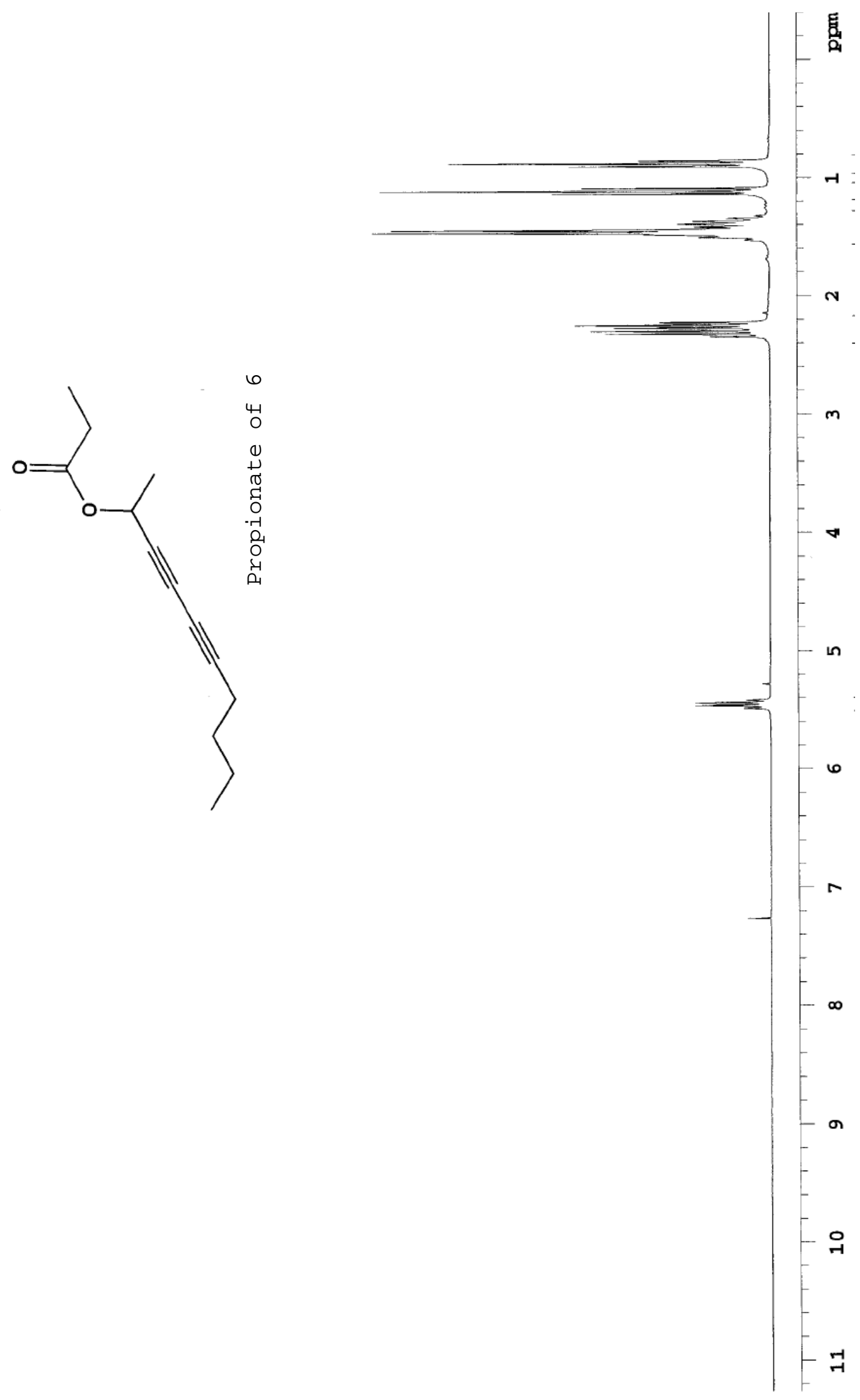




$$
1
$$




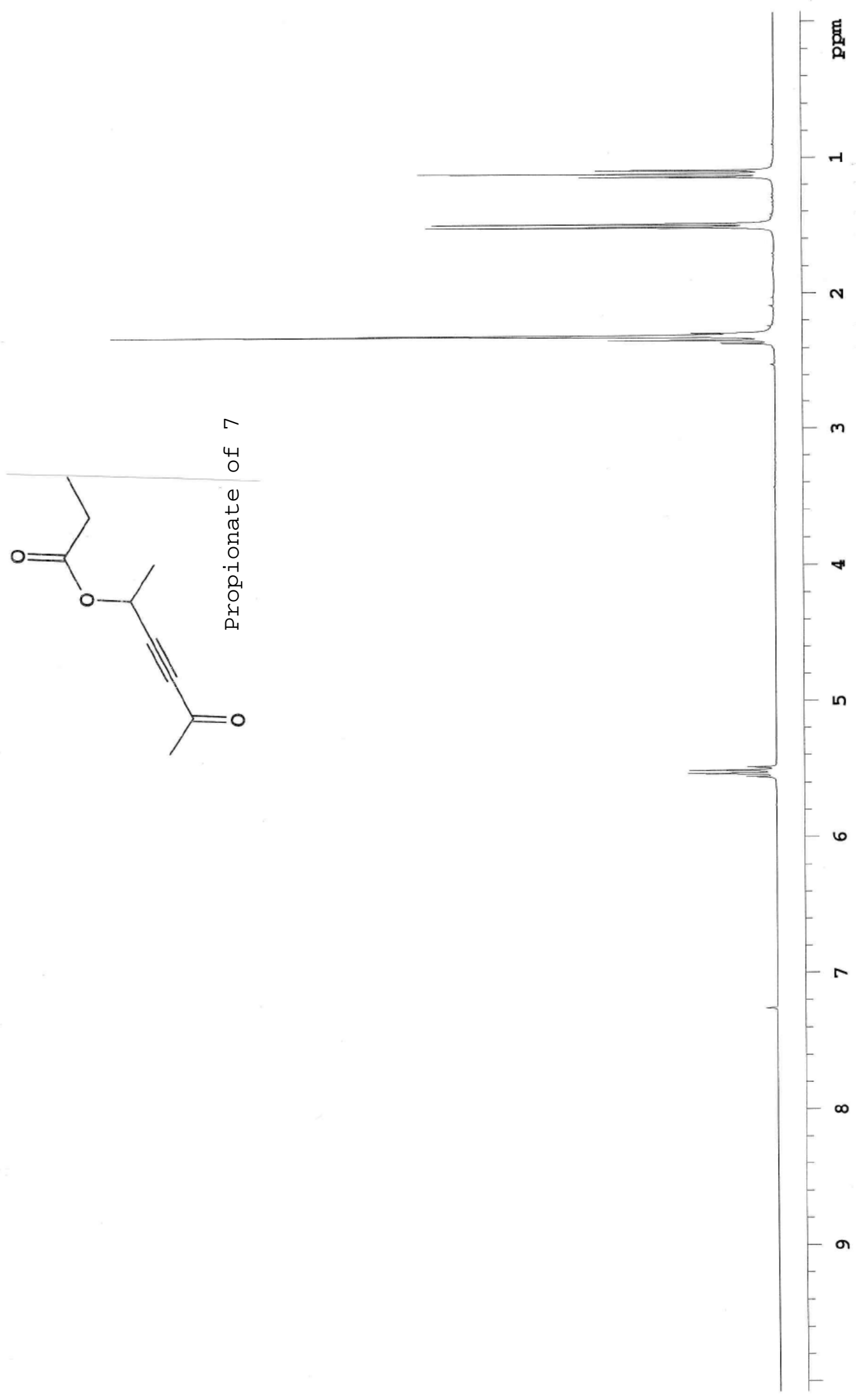



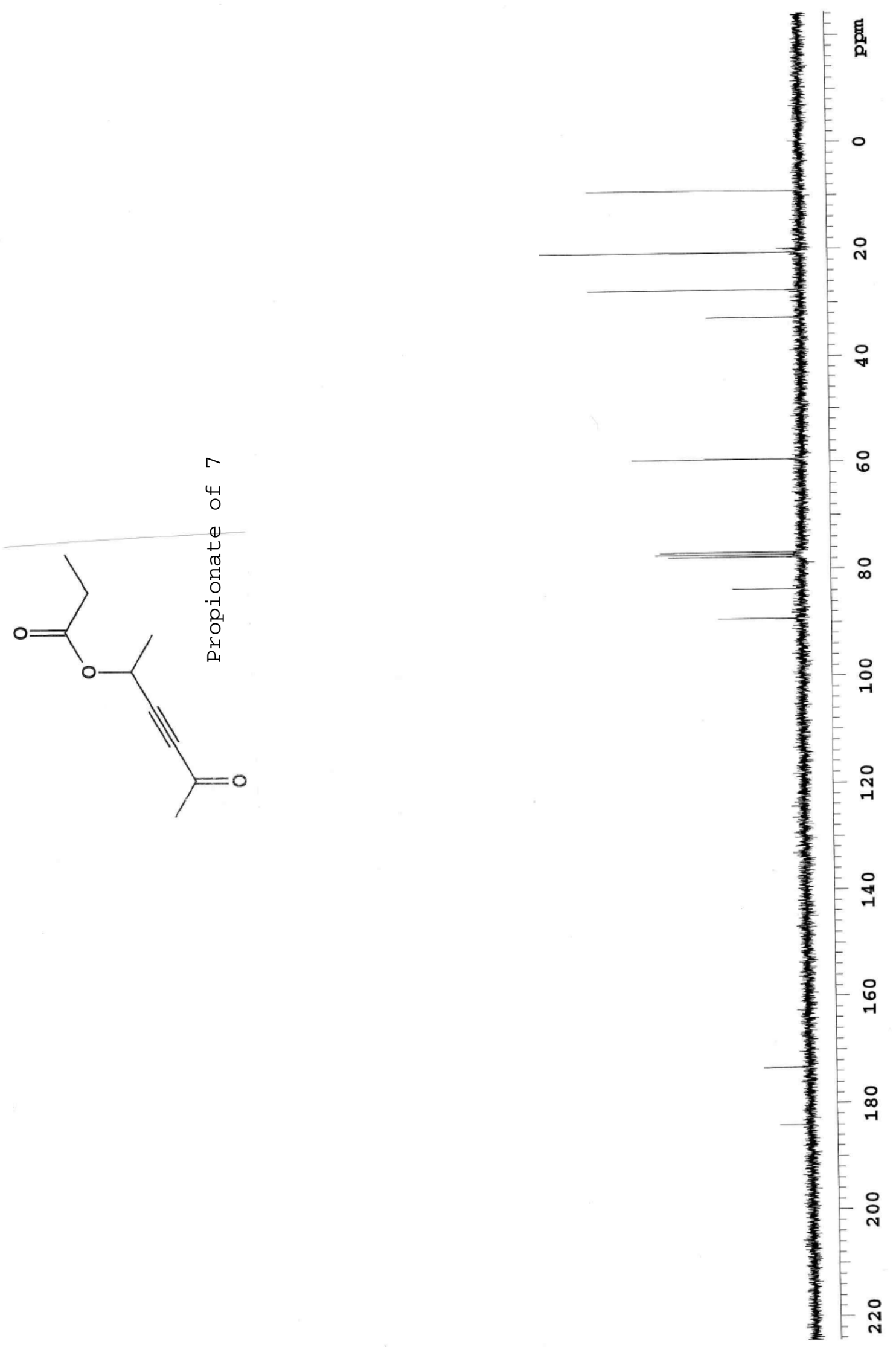

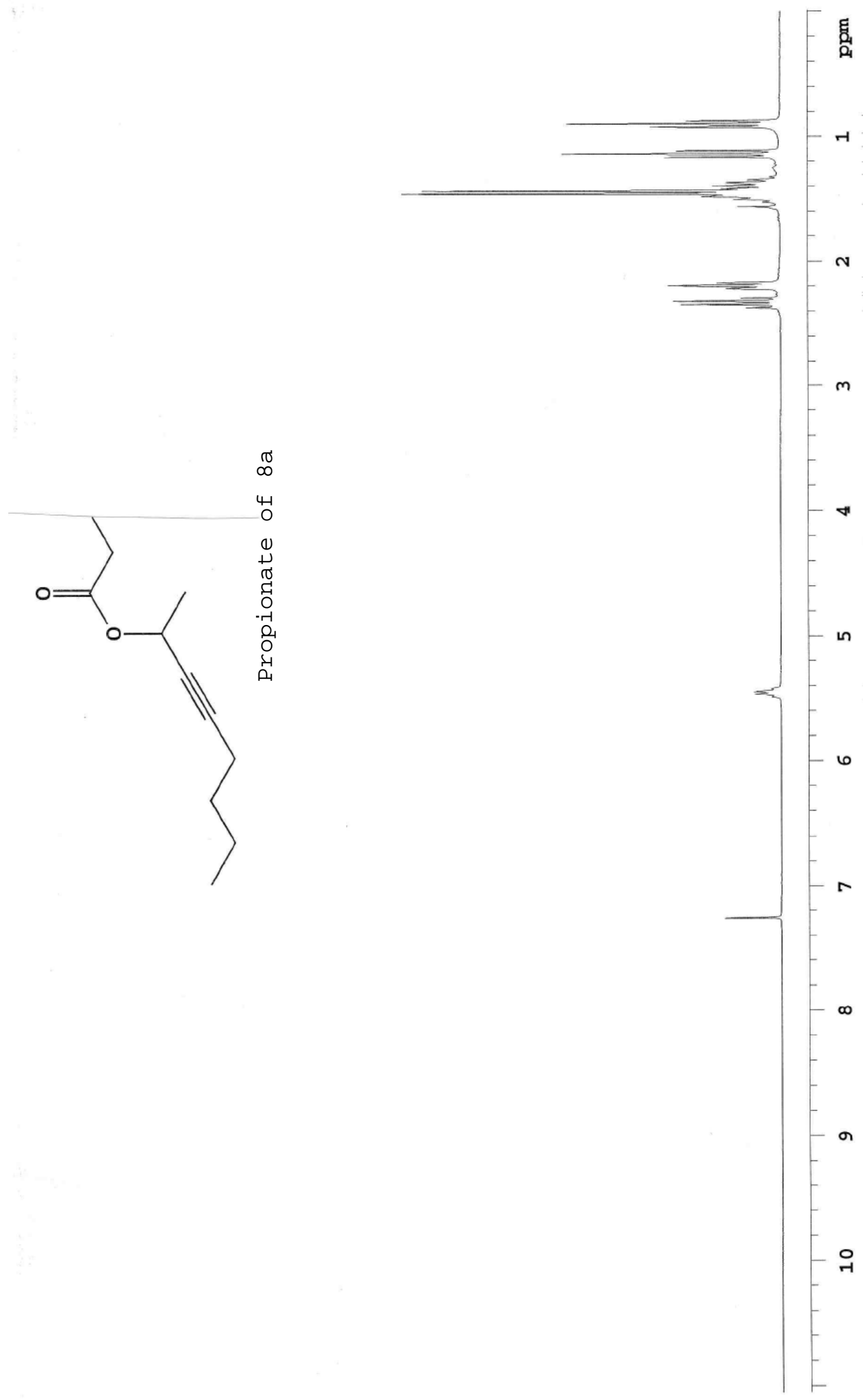

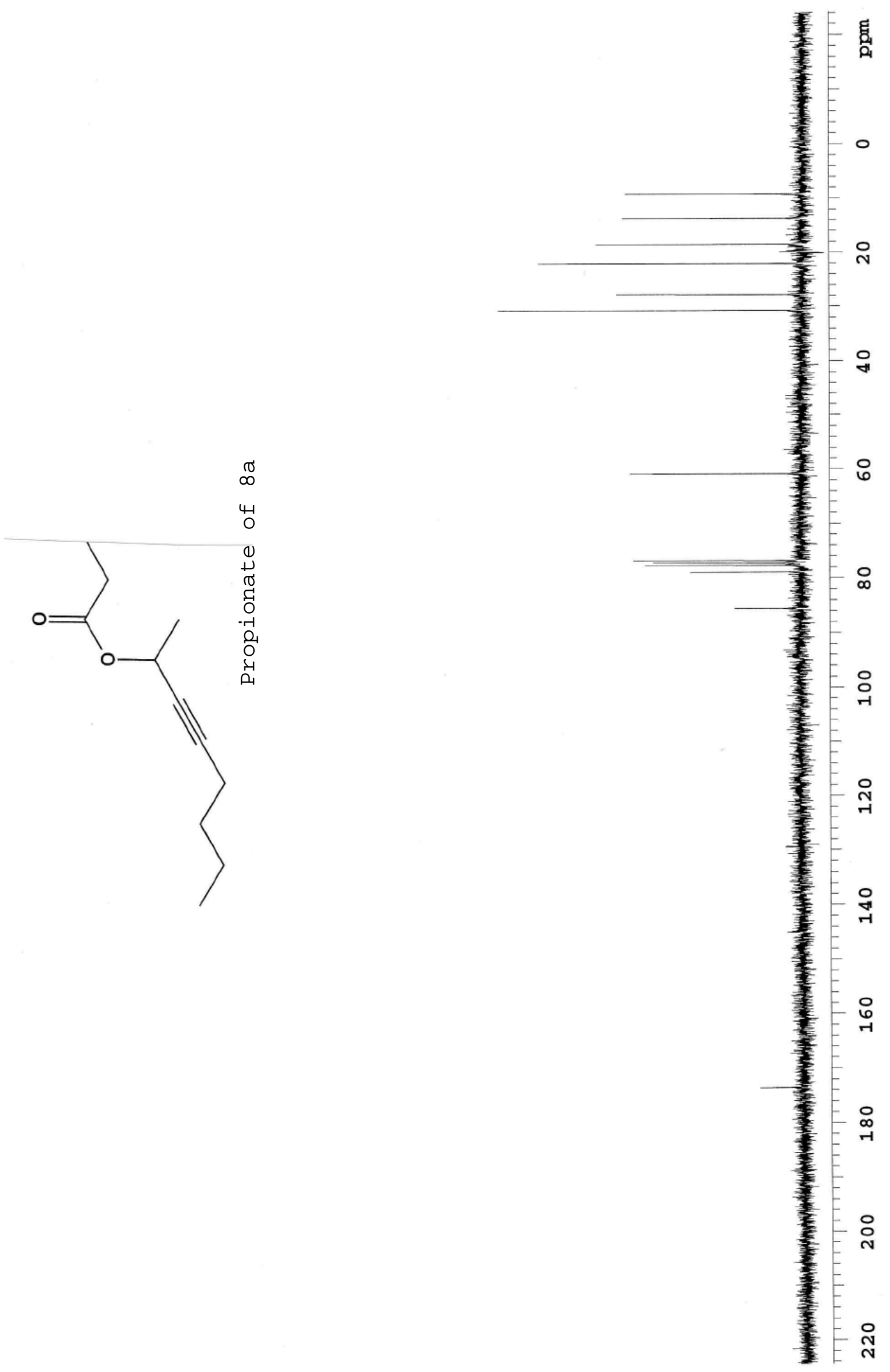


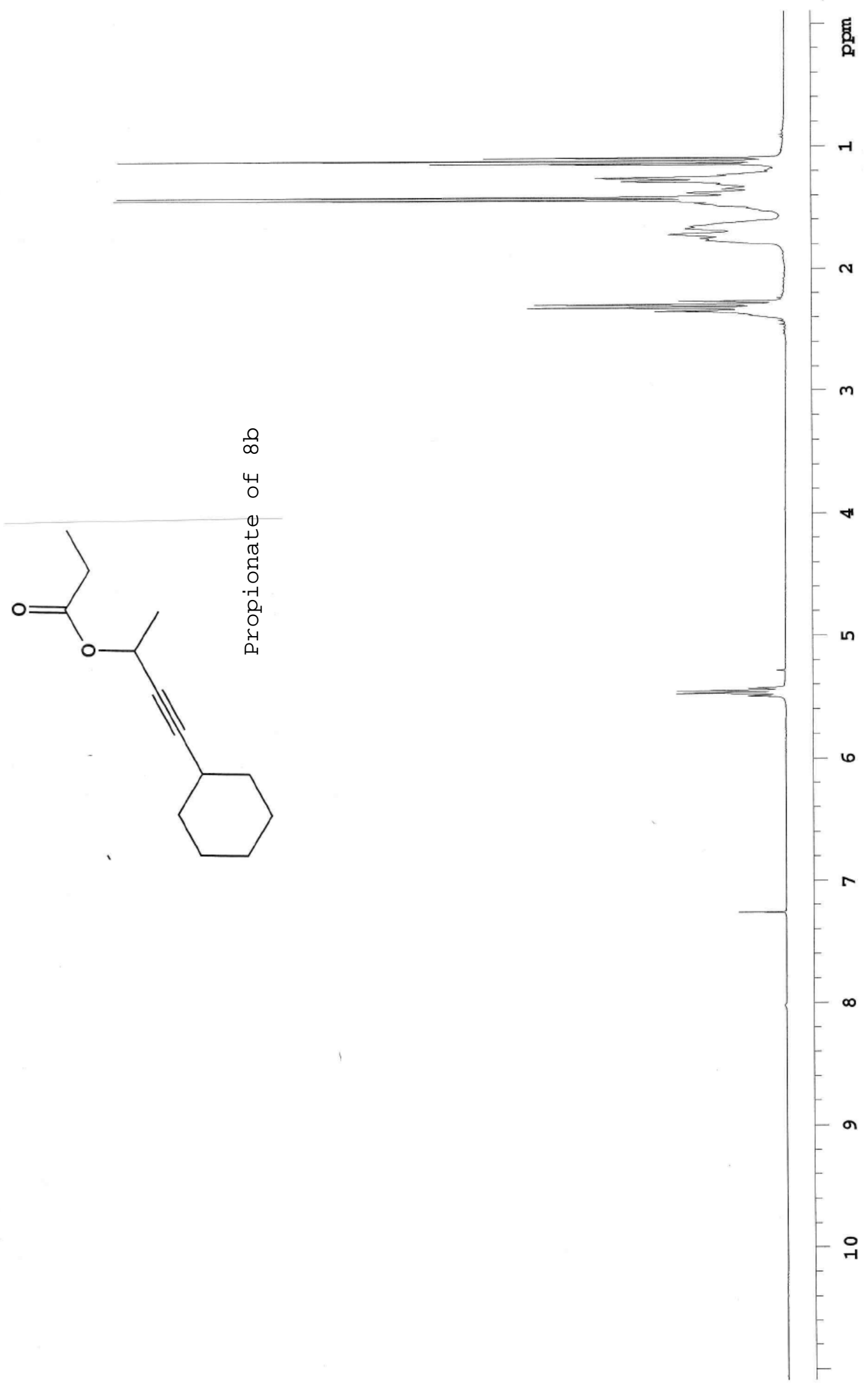




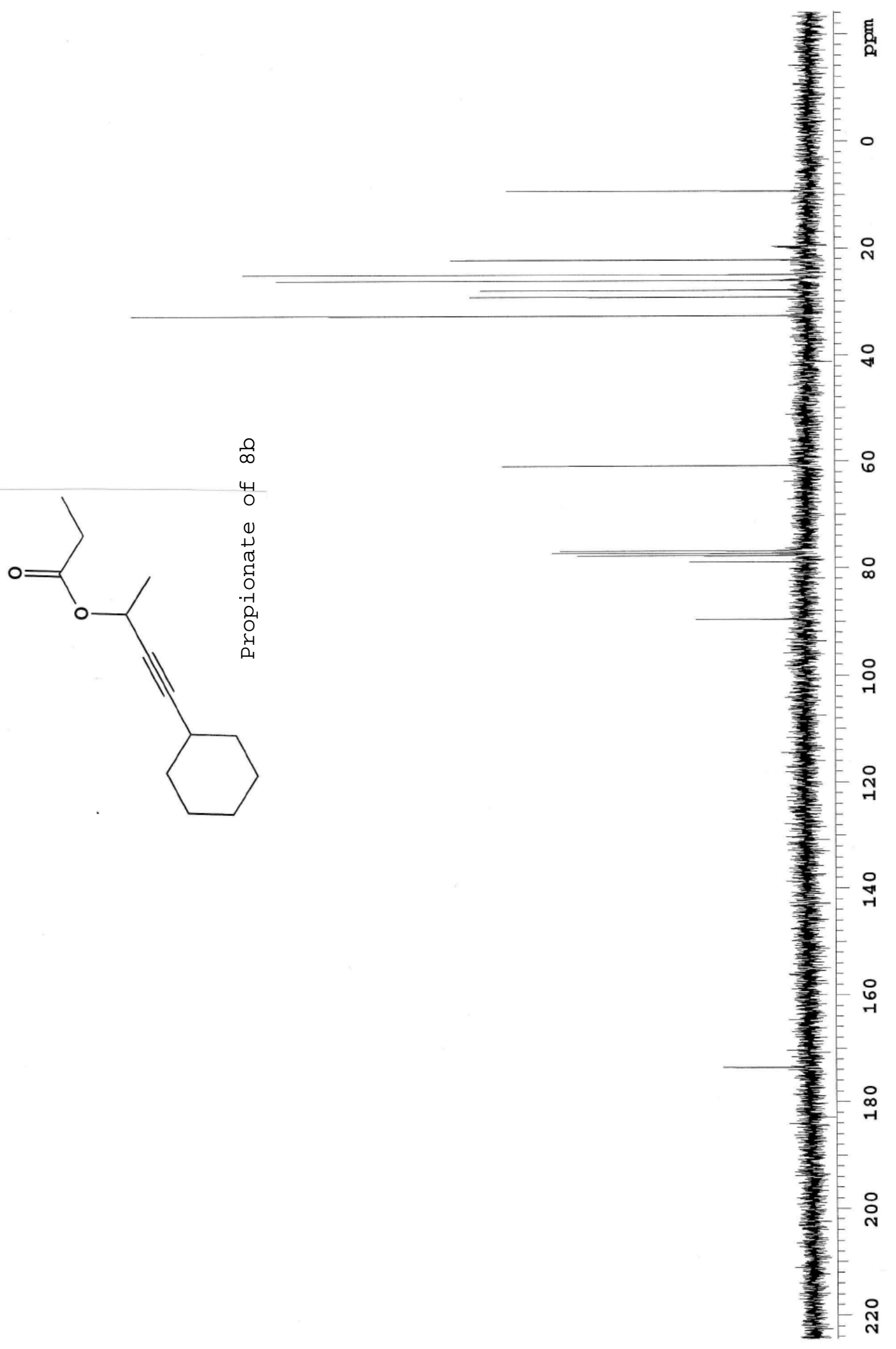

25 


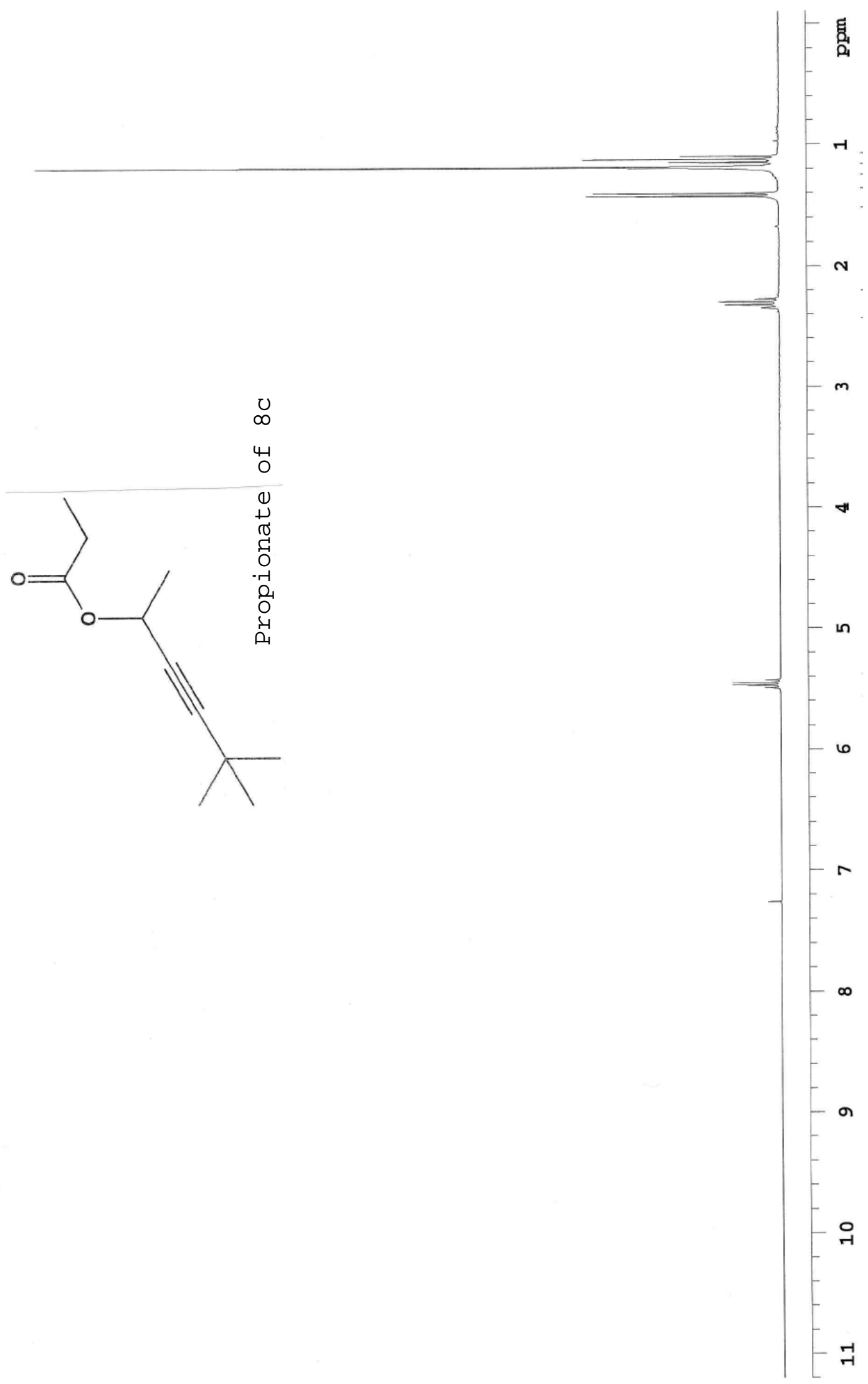




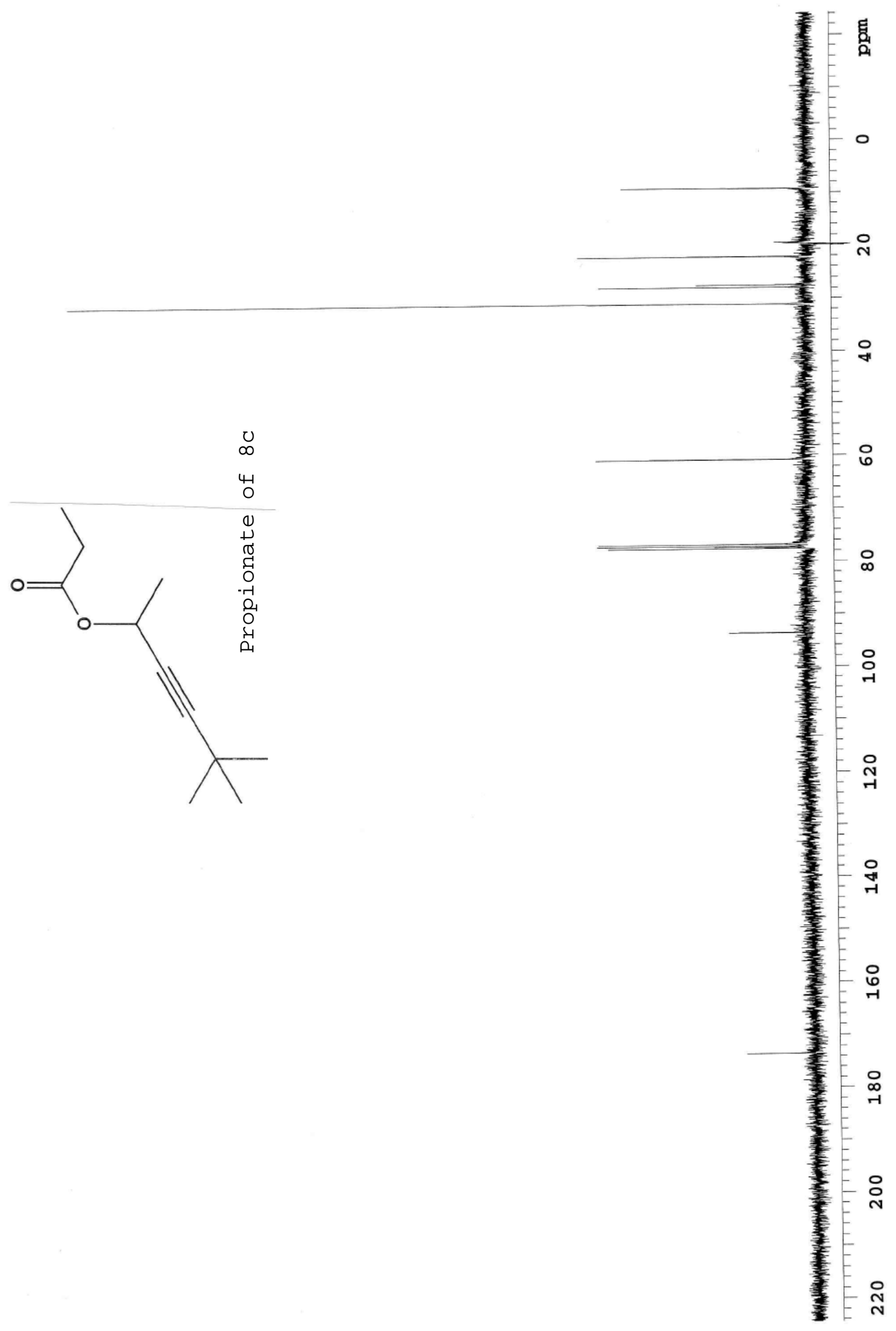




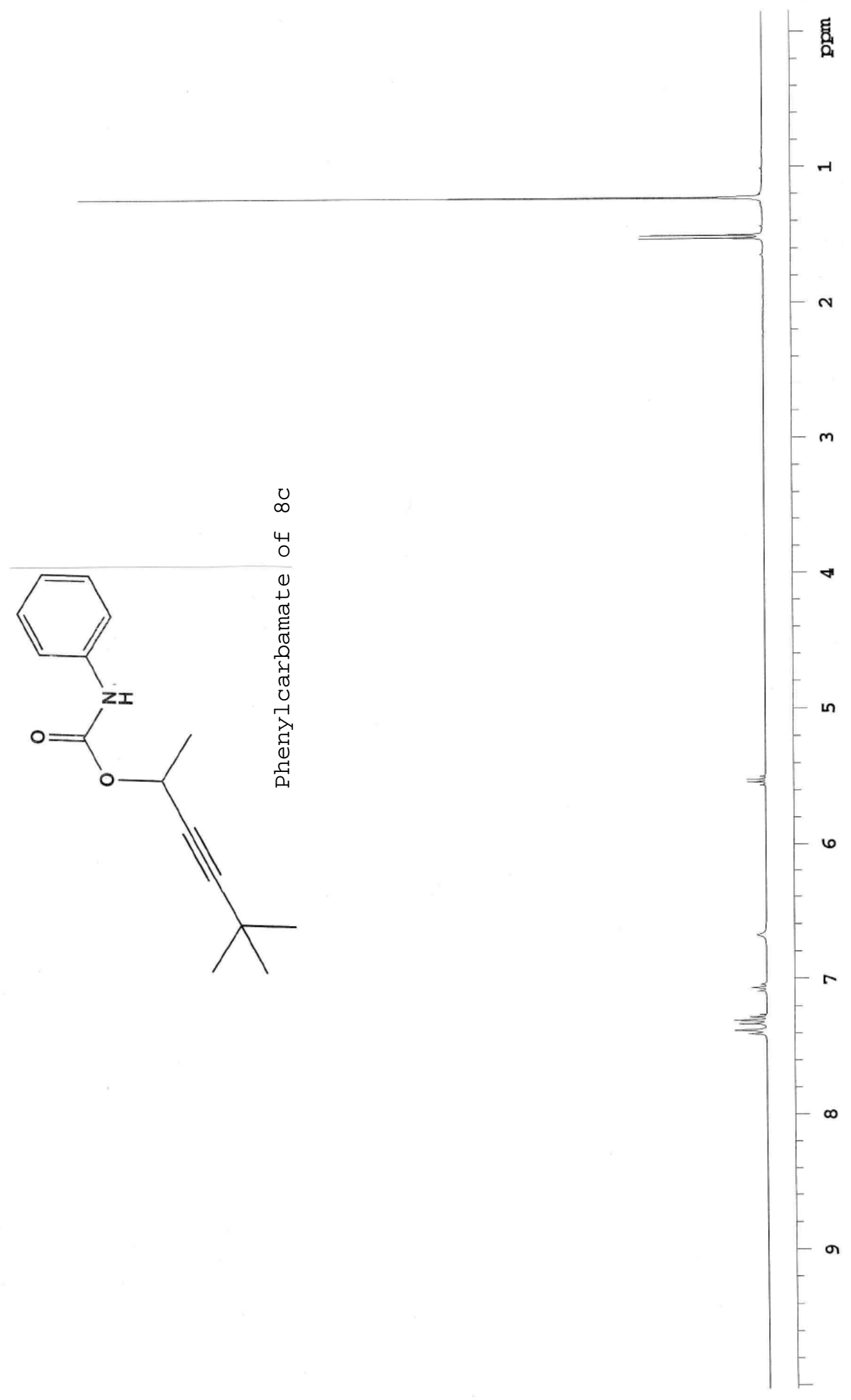




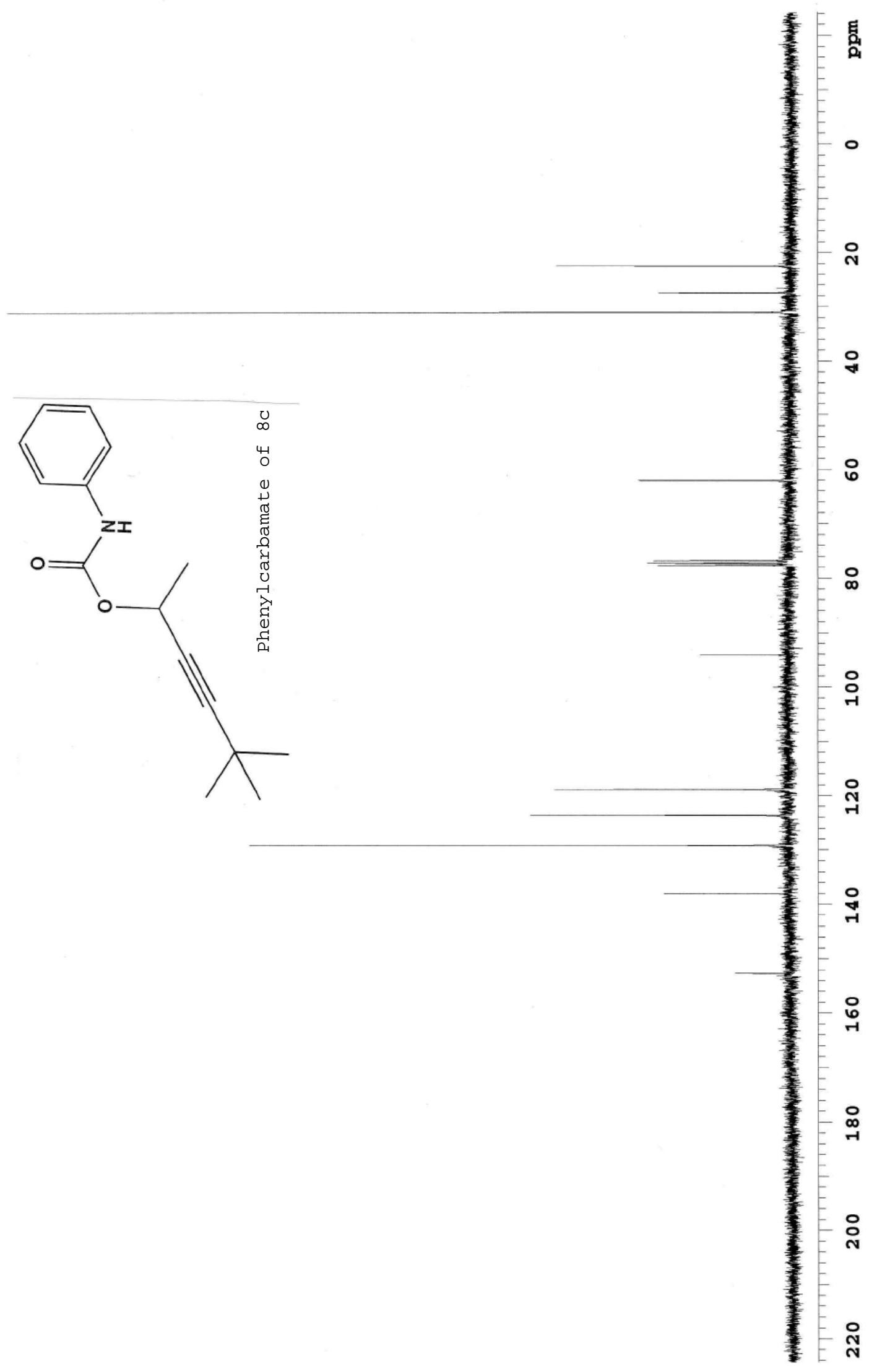




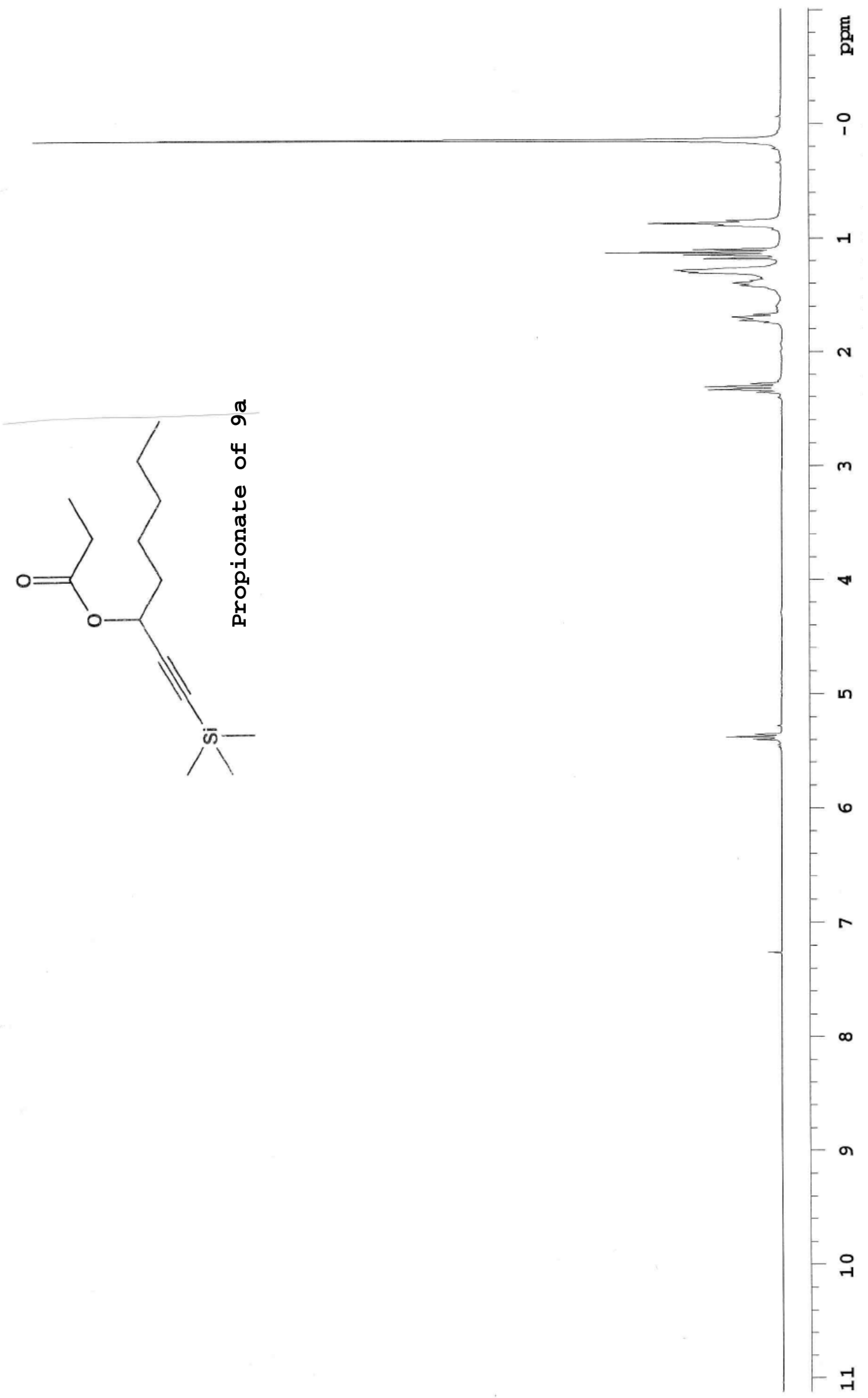



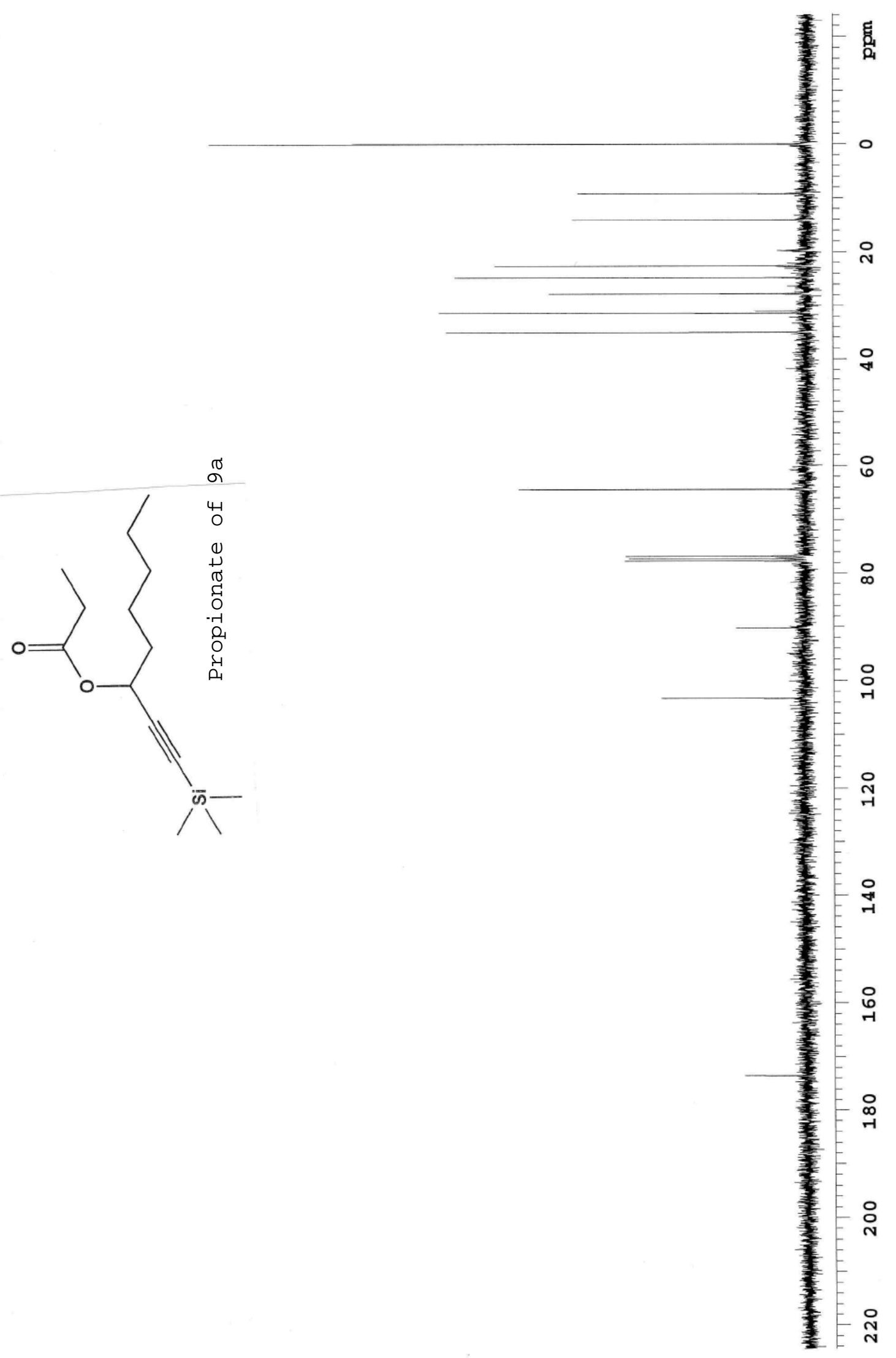